Article

\title{
Polyphenol Levels Are Inversely Correlated with Body Weight and Obesity in an Elderly Population after 5 Years of Follow Up (The Randomised PREDIMED Study)
}

\author{
Xiaohui Guo ${ }^{1}$, Anna Tresserra-Rimbau ${ }^{1,2}$, Ramón Estruch ${ }^{2,3}$, Miguel A. Martínez-González 2,4,5, \\ Alexander Medina-Remón 2,3, Montserrat Fitó ${ }^{2,6}$, Dolores Corella ${ }^{2,7}$, Jordi Salas-Salvadó ${ }^{2,8}$, \\ Maria Puy Portillo ${ }^{2,9}$, Juan J. Moreno ${ }^{1}$, Xavier Pi-Sunyer ${ }^{10}$ and Rosa M. Lamuela-Raventós ${ }^{1,2, *}$ \\ 1 Department of Nutrition, Food Science and Gastronomy, XaRTA, INSA-UB, School of Pharmacy and Food \\ Science, University of Barcelona, 08028 Barcelona, Spain; guoxiaohui1130@gmail.com (X.G.); \\ annatresserra@ub.edu (A.T.-R.); jjmoreno@ub.edu (J.J.M.) \\ 2 CIBEROBN Fisiopatología de la Obesidad y Nutrición, Instituto de Salud Carlos III, 28029 Madrid, Spain; \\ restruch@clinic.ub.es (R.E.); mamartinez@unav.es (M.A.M.-G.); amedina@ub.edu (A.M.-R.); \\ mfito@imim.es (M.F.); dolores.corella@uv.es (D.C.); jordi.salas@urv.cat (J.S.-S.); \\ mariapuy.portillo@ehu.es (M.P.P.) \\ 3 Department of Internal Medicine, Hospital Clínic, IDIBAPS, University of Barcelona, 08036 Barcelona, Spain \\ 4 Department of Preventive Medicine and Public Health, School of Medicine \& IdiSNA \\ (Institute for Health Research), University of Navarra, 31080 Pamplona, Spain \\ 5 Department of Nutrition, Harvard TH Chan School of Public Health, Boston, MA 02115, USA \\ 6 Cardiovascular Risk and Nutrition Research Group (CARIN, Regicor Study Group), IMIM \\ (Hospital del Mar Medical Research Institute), 08003 Barcelona, Spain \\ 7 Department of Epidemiology, Preventive Medicine and Public Health, School of Medicine, \\ University of Valencia, 46010 Valencia, Spain \\ 8 Human Nutrition Unit, University Hospital of Sant Joan de Reus, Department of Biochemistry and \\ Biotechnology, Faculty of Medicine and Health Sciences, IISPV, Rovira i Virgili University, 43201 Reus, Spain \\ 9 Nutrition and Obesity Group, Department of Nutrition and Food Science, Faculty of Pharmacy and \\ Lucio Lascaray Research Institute, University of País Vasco (UPV/EHU), 01006 Vitoria, Spain \\ 10 New York Obesity Research Center, Department of Medicine and Institute of Human Nutrition, \\ Columbia University, New York, NY 10032, USA; fxp1@columbia.edu \\ * Correspondence: lamuela@ub.edu; Tel.: +34-934-034-843
}

Received: 17 March 2017; Accepted: 26 April 2017; Published: 3 May 2017

\begin{abstract}
Overweight and obesity have been steadily increasing in recent years and currently represent a serious threat to public health. Few human studies have investigated the relationship between polyphenol intake and body weight. Our aim was to assess the relationship between urinary polyphenol levels and body weight. A cross-sectional study was performed with 573 participants from the PREDIMED (Prevención con Dieta Mediterránea) trial (ISRCTN35739639). Total polyphenol levels were measured by a reliable biomarker, total urinary polyphenol excretion (TPE), determined by the Folin-Ciocalteu method in urine samples. Participants were categorized into five groups according to their TPE at the fifth year. Multiple linear regression models were used to assess the relationships between TPE and obesity parameters; body weight (BW), body mass index (BMI), waist circumference (WC), and waist-to-height ratio (WHtR). After a five years follow up, significant inverse correlations were observed between TPE at the 5th year and BW ( $\beta=-1.004 ; 95 \%$ CI: -1.634 to $-0.375, p=0.002)$, BMI ( $\beta=-0.320 ; 95 \%$ CI: -0.541 to $-0.098, p=0.005)$, WC $(\beta=-0.742 ; 95 \%$ CI: -1.326 to $-0.158, p=0.013)$, and WHtR ( $\beta=-0.408$; $95 \%$ CI: -0.788 to $-0.028, p=0.036)$ after adjustments for potential confounders. To conclude, a greater polyphenol intake may thus contribute to reducing body weight in elderly people at high cardiovascular risk.
\end{abstract}


Keywords: overweight; obesity; polyphenol; urine; PREDIMED

\section{Introduction}

Overweight and obesity have been steadily increasing in recent years and currently represent a serious threat to public health [1]. In 2014, more than 1.9 billion adults were overweight worldwide, and of these over 600 million were obese [2]. With nearly three million adults dying each year as a result of being overweight or obese, the impact of obesity on morbidity, mortality, and health care costs is very high [3]. Lifestyle and dietary habits are key determinants in the prevalence of obesity [4-6].

Polyphenols, the most abundant antioxidants in nature, are widely distributed in plant-derived foods such as vegetables, fruits, seeds, coffee, wine, and tea [7]. Only a few human studies have reported a relationship between polyphenol intake and body weight, even though obesity is considered a major independent risk factor for various chronic diseases [8,9]. Evidence for the effects of polyphenols on obesity parameters in humans is inconsistent, possibly due to divergence among study designs, characteristics of the participants, and metabolic pathways. Although some intervention clinical trials with polyphenol-enriched food or polyphenol extracts do not show any effect on weight or waist circumference [10-12], other studies have reported that polyphenols reduce body weight and increase energy expenditure [13-16]. The oral bioavailability of polyphenols is particularly important because, after being modified and metabolized by enzymes, their concentration in tissues and biological fluids is quite low $[9,12,15,17]$. There is therefore a need for a biomarker to accurately reflect polyphenol concentration after their absorption and metabolism.

Polyphenol plasma levels or total urinary polyphenol excretion, considered in recent years as a reliable biomarker of total polyphenol intake, has been correlated with dietary polyphenol intake, and has been applied to explore associations between polyphenol intake and several chronic disease risk parameters [18-21]. Thus, the objective of the current study was to assess the associations between total polyphenol intake, measured by total urinary polyphenol excretion (TPE), and obesity parameters in an elderly population at high cardiovascular risk after five years of follow up.

\section{Materials and Methods}

The protocol for this trial and supporting Strengthening the Reporting of Observational studies in Epidemiology (STROBE) checklist are available as supporting information.

\subsection{Ethics Statement}

All participants provided informed consent. The Institutional Review Board (IRB) of the Hospital Clinic (Barcelona, Spain), accredited by the US Department of Health and Human Services (DHHS) update for Federal wide Assurance for the Protection of Human Subjects for International (Non-US) Institutions \#00000738, approved the study protocol on 16 July 2002. The authors confirm that all ongoing and related trials for this drug/intervention are carried out following the rules of the Declaration of Helsinki of 1975 and registered (ISRCTN35739639).

\subsection{Subjects}

Participants were drawn from the PREDIMED Study ('Prevención con Dieta Mediterránea' (Prevention with the Mediterranean Diet), ISRCTN35739639). The information in the registry was delayed after recruitment began, to be sure about the feasibility of the study protocol; we started the trial as a 'pilot study' on October 2003, and, once we were sure that the intervention protocol worked, we decided to submit the study protocol for registration (date of application: 2 September 2005). The protocol and recruitment method are reported in detail elsewhere [22]. 
The present study looks at 573 participants that were recruited in two centers, the Clinic Hospital of Barcelona and the University of Valencia, both in Spain, and all were followed-up after more than five years. The period of recruitment was from 2003 to 2006, and the average follow up was 5.9 years.

\subsection{Nutritional Measurements}

The selected participants were asked to complete some questionnaires: a validated 137-item food frequency questionnaire (FFQ) to assess dietary habits [23]; a 47-item general questionnaire aimed to summarize information about lifestyle, health condition, education, history of illnesses, and medication use; a 14 point questionnaire evaluating the degree of adherence to the Mediterranean diet [24]; and a validated Spanish version of the Minnesota Leisure-Time Physical Activity Questionnaire to record physical activity [25]. Nutrient intake was adjusted by calories using the residual method [26]. All questionnaires were administered and repeated yearly during the follow up by trained staff in face-to-face interviews.

\subsection{Urine Samples}

Spot urine samples from the participants were collected and coded at the clinic by a technician and then immediately shipped to a central laboratory to be stored at $-80^{\circ} \mathrm{C}$ until analyzed.

\subsection{TPE Measurements}

The Folin-Ciocalteu method was applied to determine the content of TPE, using a clean-up procedure with solid phase extraction (SPE) performed in 96-well plate cartridges (Oasis MAX), which helped to remove urinary interferences. Finally, TPE was expressed as milligrams of gallic acid equivalent (GAE)/g of creatinine. All details have been previously described by Medina-Remón et al. [19].

\subsection{Measurements}

Weight and height were measured with calibrated scales and a wall-mounted stadiometer, respectively. Body mass index (BMI) was calculated as weight in kilograms divided by the square of height in meters. Waist circumference (WC) was measured midway between the lowest rib and the iliac crest. Waist-to-height ratio (WHtR) was calculated as the waist in centimeters divided by the height in meters. Blood pressure was determined in triplicate using a validated semi-automatic sphygmomanometer (Omron HEM-705CP, Tokyo, Japan) by trained nurses. Measurements were taken at three time points, separated by $2 \mathrm{~min}$, while the participant was in a seated position after $5 \mathrm{~min}$ of rest [27]. Obesity is defined as BMI more than $30 \mathrm{~kg} / \mathrm{m}^{2}$.

\subsection{Statistical Analysis}

Results were expressed as mean $\pm \mathrm{SD}$ for continuous variables or percentages for categorical variables. Kolmogorov and Levene tests were applied to examine the normality distribution and skewness. All participants, including total subjects, males, and females were divided into five categories according to their TPE at the fifth year of follow up. Changes in nutrient intakes and key food consumption according to the FFQs were assessed with yearly repeated-measures analysis during the follow up period. A Bonferroni post-hoc test and paired $t$-test were used to compare each variable within and between groups.

Multiple linear regression models were used to assess the relationship between anthropometric parameters (Body weight (BW), BMI, WC, and WHtR) and quintiles of TPE at the fifth year, adjusted for potential confounders, including sex, age, intervention groups, smoking status (never, current, former), family history of coronary heart disease (CHD), physical activity, hypertension, diabetes, dyslipidemia, marital status (single, married, widowed), education level (primary school, high school, university), medication used (antihypertensive drugs, vitamins, insulin, oral hypoglycemic drugs, 
aspirin, or other antiplatelet drug supplements taken in the last month), recruitment centers, 14 unit Mediterranean diet score, and energy intake at baseline. Multiple logistic regression analyses were used to calculate the odds ratio (OR) for quintiles of TPE and obesity (BMI $>30 \mathrm{~kg} / \mathrm{m}^{2}$ ). Models were adjusted for potential confounders as in linear regression analyses.

All analyses were performed using SPSS software V21.0 (SPSS Inc., Chicago, IL, USA,). All models were tested for the detection of outliers, multicollinearity, homoscedasticity, and normality and independence of errors. All statistical tests were two-tailed, and the significance level was $p<0.05$. The detailed information of the participants is available as supporting information.

\section{Results}

A total of 650 subjects were randomly selected from two centers, the Hospital Clinic of Barcelona and the University of Valencia. From them, 38 were excluded because they did not meet the inclusion criteria during the intervention, and, after five years, 39 were excluded because their TPE concentrations were considered outliers, which was defined as any data point more than 1.5 interquartile ranges below the first quartile or above the third quartile; hence a total of 573 participants were finally included (Figure 1).

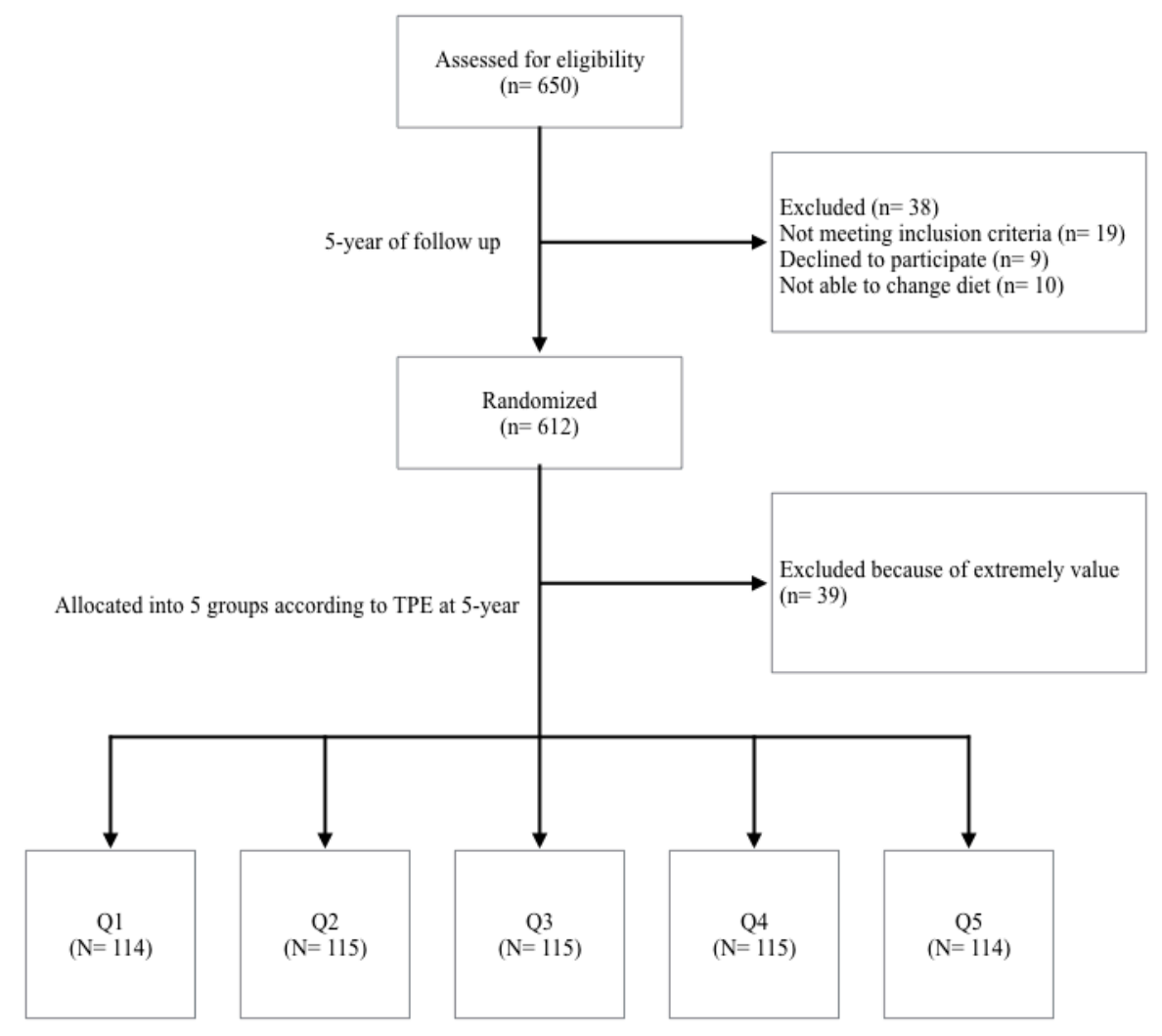

Figure 1. Flowchart of study participants.

The baseline characteristics of participants grouped by quintiles of TPE at baseline are shown in Table 1. There were a total of 277 men and 296 women with a mean age of $66.2 \pm 6.1$ years and $68.3 \pm 5.4$ years, respectively. Of those participants, $41.5 \%$ had diabetes, $80.5 \%$ had hypertension, $66.8 \%$ had dyslipidemia, $16.9 \%$ were current smokers, and $37.5 \%$ had a family history of CHD. Compared with participants with the lowest TPE, those with higher TPE were more likely to be women, older, less likely to smoke, and also had lower body weight. Q4 shows the lowest prevalence of hypertension. 
Table 1. Baseline characteristics of participants according to quintiles of total urinary polyphenol excretion (TPE) at baseline.

\begin{tabular}{|c|c|c|c|c|c|c|c|c|c|c|c|}
\hline & \multicolumn{11}{|c|}{ TPE (mg GAE/g Creatinine) } \\
\hline & \multicolumn{2}{|c|}{ Q1 } & \multicolumn{2}{|c|}{ Q2 } & \multicolumn{2}{|c|}{ Q3 } & \multicolumn{2}{|c|}{ Q4 } & \multicolumn{2}{|c|}{ Q5 } & \multirow{2}{*}{$p$} \\
\hline & \multicolumn{2}{|c|}{$(<76.55)$} & \multicolumn{2}{|c|}{$(76.56-95.20)$} & \multicolumn{2}{|c|}{$(95.21-119.18)$} & \multicolumn{2}{|c|}{ (119.19-145.86) } & \multicolumn{2}{|c|}{$(>145.86)$} & \\
\hline No. of subjects & 114 & - & 115 & - & 115 & - & 115 & - & 114 & - & - \\
\hline Women, $n(\%)$ & 33 & 28 & 49 & 42.6 & 66 & 57.4 & 65 & 56.5 & 83 & 72.8 & $<0.001$ \\
\hline Age $(y)$, mean $(S D)$ & 66.4 & 5.9 & 66.6 & 6.0 & 66.9 & 5.9 & 67.7 & 5.7 & 68.9 & 5.8 & 0.007 \\
\hline Weight $(\mathrm{kg})$, mean (SD) & 80.2 & 11.8 & 77.7 & 10.4 & 73.7 & 9.1 & 73.3 & 10.9 & 70.8 & 10.4 & $<0.001$ \\
\hline $\operatorname{BMI}\left(\mathrm{kg} / \mathrm{m}^{2}\right)$, mean (SD) & 29.8 & 2.9 & 29.5 & 3.2 & 29.1 & 3.1 & 28.9 & 3.6 & 28.8 & 3.4 & 0.080 \\
\hline Systolic BP (mm Hg), mean (SD) & 150.9 & 16.9 & 153.9 & 19.5 & 149.9 & 17.0 & 151.8 & 19.5 & 150.4 & 15.9 & 0.454 \\
\hline Diastolic BP (mm Hg), mean (SD) & 86.4 & 10.5 & 86.1 & 10.4 & 85.0 & 9.9 & 84.2 & 10.3 & 84.4 & 9.1 & 0.363 \\
\hline Hypertension, $n(\%)$ & 93 & 81.6 & 102 & 88.7 & 94 & 81.7 & 79 & 68.7 & 93 & 81.6 & 0.004 \\
\hline Diabetes, $n(\%)$ & 47 & 41.2 & 41 & 35.7 & 48 & 41.7 & 53 & 46.1 & 49 & 43.0 & 0.605 \\
\hline Dyslipidemia, $n(\%)$ & 72 & 63.2 & 74 & 64.3 & 78 & 67.8 & 80 & 69.6 & 79 & 69.3 & 0.779 \\
\hline Smoking status, $n(\%)$ & & & & & & & & & & & 0.002 \\
\hline Current & 32 & 28.1 & 22 & 19.1 & 16 & 13.9 & 18 & 15.7 & 9 & 7.9 & - \\
\hline Former & 26 & 22.8 & 25 & 21.7 & 24 & 20.9 & 31 & 27.0 & 20 & 17.5 & - \\
\hline Never & 56 & 49.1 & 68 & 59.1 & 75 & 65.2 & 66 & 57.4 & 85 & 74.6 & - \\
\hline Family history of CHD, $n(\%)$ & 39 & 34.2 & 42 & 36.5 & 45 & 39.1 & 41 & 35.7 & 48 & 42.1 & 0.95 \\
\hline Medication, $n(\%)$ & & & & & & & & & & & - \\
\hline Aspirin & 21 & 18.4 & 17 & 14.8 & 27 & 23.5 & 19 & 16.5 & 19 & 16.7 & 0.482 \\
\hline Antihypertensive drugs & 87 & 76.3 & 90 & 78.3 & 82 & 71.3 & 71 & 61.7 & 84 & 73.7 & 0.049 \\
\hline Hypolipidemic drugs & 40 & 35.1 & 45 & 39.1 & 50 & 43.5 & 53 & 46.1 & 51 & 44.7 & 0.426 \\
\hline Insulin & 3 & 2.6 & 7 & 6.1 & 4 & 3.5 & 7 & 6.1 & 6 & 5.3 & 0.639 \\
\hline Oral hypoglycemic drugs & 23 & 20.2 & 22 & 19.1 & 29 & 25.2 & 30 & 26.1 & 27 & 23.7 & 0.652 \\
\hline Vitamin or minerals & 5 & 4.4 & 5 & 4.3 & 10 & 8.7 & 9 & 7.8 & 18 & 15.8 & 0.005 \\
\hline Education level, $n(\%)$ & & & & & & & & & & & 0.348 \\
\hline University & 13 & 11.4 & 16 & 13.9 & 10 & 8.7 & 7 & 6.1 & 9 & 7.9 & - \\
\hline High school & 21 & 18.4 & 14 & 12.2 & 18 & 15.7 & 22 & 19.1 & 13 & 11.4 & - \\
\hline Primary school & 79 & 69.3 & 83 & 72.2 & 85 & 73.9 & 86 & 74.8 & 92 & 80.7 & - \\
\hline Marital status, $n(\%)$ & & & & & & & & & & & 0.168 \\
\hline Single & 7 & 6.1 & 5 & 4.3 & 4 & 3.5 & 3 & 2.6 & 6 & 5.3 & - \\
\hline Married & 96 & 84.2 & 93 & 80.9 & 93 & 80.9 & 88 & 76.5 & 80 & 70.2 & - \\
\hline Widowed & 10 & 8.8 & 14 & 12.2 & 16 & 13.9 & 24 & 20.9 & 25 & 21.9 & - \\
\hline $\begin{array}{l}\text { Physical activity at leisure time } \\
\text { (MET-min/d), mean (SD) }\end{array}$ & 267.5 & 222.5 & 302.7 & 256.3 & 233 & 172.9 & 283.7 & 271.3 & 261.7 & 247.3 & 0.237 \\
\hline
\end{tabular}

TPE: total polyphenol excretion; GAE: gallic acid equivalent; BMI: body mass index; BP: blood pressure; CHD: coronary heart diseases. Data are given as means (SD) for continuous variables and percentages for categorical variables; $p<0.05$ indicates statistical significance. * $p$-values calculated by analysis of variance or $\chi^{2}$ tests. 
The comparison of total urinary polyphenol excretion between baseline and the fifth year of follow up by quintiles of TPE at the fifth year is shown in Figure 2. For the first two quintiles, TPE at baseline was significantly higher than at the fifth year. By contrast, TPE at the top two categories was higher than at baseline.

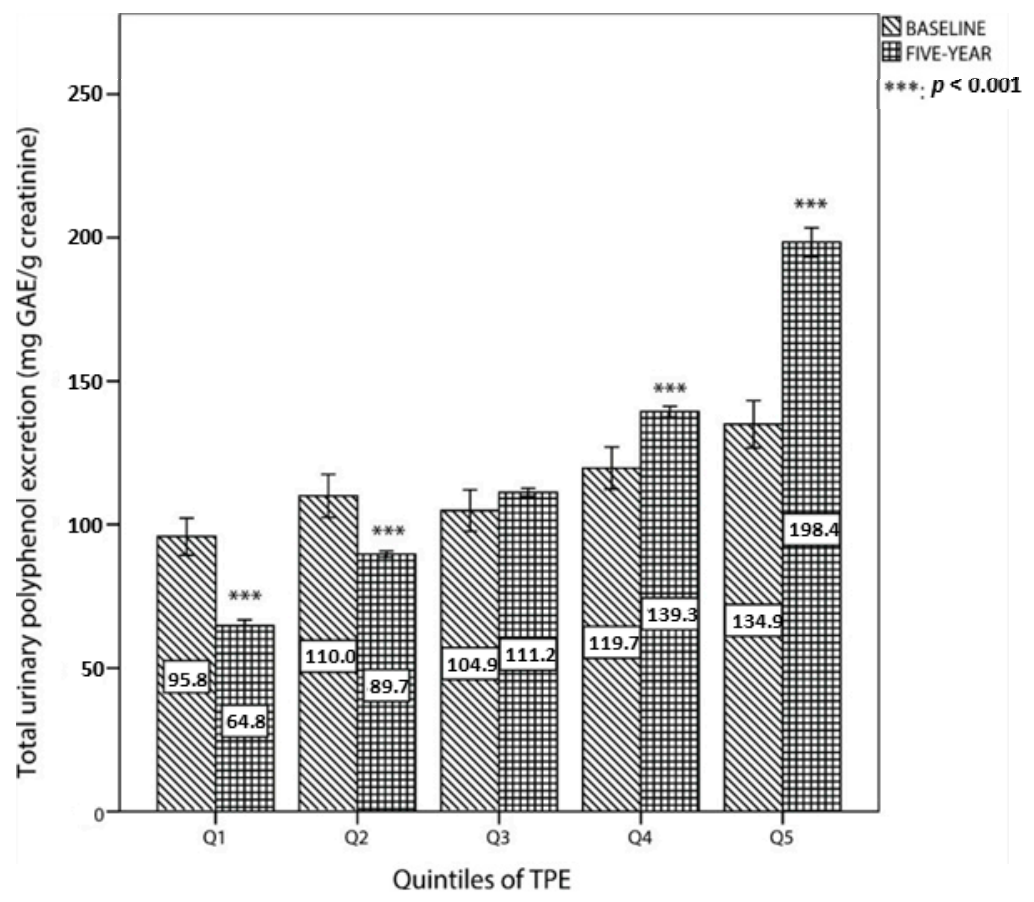

Figure 2. Total polyphenol excretion at baseline and at the fifth year of follow up by quintiles of TPE.

Table 2 summarizes information on key changes in food consumption during the intervention according to quintiles of TPE. As shown, at the end of the intervention, the consumption of most of the items belonging to a Mediterranean dietary pattern had increased significantly, including vegetables, fruits, fish, extra virgin olive oil, olive oil, nuts, coffee, and milk. However, the intake of wine decreased significantly, as well as intakes of cereals, meat, and pastries. Table 2 also shows changes in nutrient intake and degrees of adherence to a Mediterranean diet. Significant increments were observed in the consumption of total fat, fiber, polyunsaturated fatty acids (PUFA), monounsaturated fatty acids (MUFA), folic acid, potassium (K), and magnesium (Mg), while total carbohydrates, protein, cholesterol, sodium ( $\mathrm{Na}$ ), and saturated fatty acids (SFA) remained similar throughout. 
Table 2. Changes in key food intake and nutrients according to the food frequency questionnaires (FFQs) after energy adjustment categorized by quintile of TPE at the fifth year ${ }^{\mathrm{a}}$.

\begin{tabular}{|c|c|c|c|c|c|c|c|c|c|c|c|c|c|c|c|}
\hline & & \multicolumn{14}{|c|}{ TPE (mg GAE/g Creatinine) } \\
\hline & & \multirow{2}{*}{\multicolumn{2}{|c|}{$\begin{array}{c}\text { Q1 } \\
(<79.02)\end{array}$}} & \multirow{2}{*}{\multicolumn{2}{|c|}{$\begin{array}{c}\text { Q2 } \\
(79.03-99.50)\end{array}$}} & \multirow{2}{*}{\multicolumn{2}{|c|}{$\begin{array}{c}\mathrm{Q3} \\
(99.51-124.53)\end{array}$}} & \multirow{2}{*}{\multicolumn{2}{|c|}{$\begin{array}{c}\mathrm{Q} 4 \\
(124.54-160.06)\end{array}$}} & \multirow{2}{*}{\multicolumn{2}{|c|}{$\begin{array}{c}\text { Q5 } \\
(>160.07)\end{array}$}} & \multirow{3}{*}{$\begin{array}{c}p^{\mathrm{b}} \\
\text { ANOVA }\end{array}$} & \multirow{2}{*}{\multicolumn{3}{|c|}{$p^{\mathrm{c}}$}} \\
\hline & & & & & & & & & & & & & & & \\
\hline & & Mean & SD & Mean & SD & Mean & SD & Mean & SD & Mean & SD & & TIME & GROUP & INTERACTION \\
\hline \multirow[t]{2}{*}{ Vegetables (g/day) } & baseline & 302.6 & 126.5 & 295.2 & 122.6 & 283.3 & 114.1 & 312.9 & 142.4 & 297.0 & 107.0 & 0.484 & $<0.001$ & 0.916 & 0.440 \\
\hline & changes & $47.7^{* *}$ & 131.5 & $53.1^{* *}$ & 130.2 & $76.3^{* *}$ & 150.4 & $46.0^{* *}$ & 162.3 & $59.0 * *$ & 131.6 & 0.514 & & & \\
\hline \multirow[t]{2}{*}{ Fruits (g/day) } & baseline & 328.9 & 184.8 & 361.6 & 179.7 & 358.6 & 182.3 & 389.5 & 162.5 & 394.5 & 189.3 & 0.064 & $<0.001$ & 0.051 & 0.530 \\
\hline & changes & $89.1 * *$ & 220.1 & $82.9 * *$ & 222.7 & $111.0^{* *}$ & 220.6 & $80.6^{* *}$ & 208.4 & $67.9 * *$ & 194.8 & 0.658 & & & \\
\hline \multirow[t]{2}{*}{ Legumes (g/day) } & baseline & 18.5 & 8.4 & 19.3 & 7.4 & 20.0 & 9.2 & 19.2 & 7.2 & 19.0 & 6.6 & 0.634 & 0.446 & 0.251 & 0.045 \\
\hline & changes & 0.3 & $\begin{array}{l}0.8 \\
10.7\end{array}$ & 1.7 & 11.3 & -3.2 & 29.1 & -1.0 & 8.5 & 1.0 & 8.3 & 0.160 & & & \\
\hline \multirow[t]{2}{*}{ Cereals (g/day) } & baseline & 246.8 & 83.8 & 246.3 & 81.2 & 232.8 & 74.2 & 242.9 & 71.9 & 237.7 & 61.9 & 0.636 & $<0.001$ & 0.530 & 0.386 \\
\hline & changes & $-22.2 *$ & 91.1 & $-21.7^{* *}$ & 85.1 & -14.5 & 91.5 & $-31.4^{* *}$ & 80.1 & $-25.6^{* *}$ & 77.5 & 0.673 & & & \\
\hline \multirow[t]{2}{*}{ Milk (g/day) } & baseline & 322.8 & 191.3 & 370.1 & 202.2 & 365.8 & 210.0 & 354.5 & 203.7 & 422.5 & 231.6 & 0.010 & 0.005 & 0.009 & 0.728 \\
\hline & changes & 38.3 & 207.4 & 21.4 & 200.9 & 10.3 & 198.6 & 40.2 * & 188.3 & 20.6 & 208.9 & 0.776 & & & \\
\hline \multirow[t]{2}{*}{ Meat (g/day) } & baseline & 138.1 & 50.4 & 136.2 & 43.9 & 145.6 & 56.5 & 139.3 & 41.7 & 141.6 & 47.1 & 0.613 & $<0.001$ & 0.198 & 0.983 \\
\hline & changes & -9.9 & 52.1 & $-13.9^{* *}$ & 44.6 & $-12.1 *$ & 52.5 & $-13.8^{* *}$ & 51.5 & $-11.7^{*}$ & 47.7 & 0.975 & & & \\
\hline \multirow[t]{2}{*}{ Fish (g/day) } & baseline & 92.0 & 39.6 & 92.5 & 36.9 & 90.4 & 38.0 & 97.0 & 41.3 & 93.0 & 39.0 & 0.787 & 0.005 & 0.970 & 0.481 \\
\hline & changes & $8.2^{*}$ & 40.4 & 6.2 & 42.2 & $11.5^{* *}$ & 44.2 & 4.2 & 39.4 & 7.3 & 43.3 & 0.764 & & & \\
\hline \multirow[t]{2}{*}{ Pastries (g/day) } & baseline & 29.6 & 32.1 & 23.6 & 22.4 & 24.1 & 24.7 & 23.9 & 22.4 & 26.6 & 26.5 & 0.379 & 0.006 & 0.291 & 0.920 \\
\hline & changes & -4.8 & 32.4 & -3.0 & 29.4 & -4.3 & 27.2 & -3.2 & 24.3 & -3.9 & 29.2 & 0.991 & & & \\
\hline \multirow[t]{2}{*}{ EVOO (g/day) } & baseline & 22.7 & 25.6 & 20.5 & 22.4 & 22.6 & 23.6 & 22.9 & 23.7 & 22.8 & 23.3 & 0.960 & $<0.001$ & 0.961 & 0.626 \\
\hline & changes & $24.9^{* *}$ & 29.6 & $27.6^{* *}$ & 27.6 & $25.4^{* *}$ & 28.2 & $26.4^{* *}$ & 30.1 & $26.9^{* *}$ & 25.6 & 0.955 & & & \\
\hline \multirow[t]{2}{*}{ Olive oil (g/day) } & baseline & 45.4 & 17.6 & 46.3 & 14.2 & 45.3 & 13.5 & 46.3 & 14.5 & 44.2 & 15.3 & 0.791 & $<0.001$ & 0.575 & 0.161 \\
\hline & changes & $7.8^{* *}$ & 18.2 & $10.5^{* *}$ & 17.5 & $9.8^{* *}$ & 16.8 & $9.3^{* *}$ & 18.7 & $11.2^{* *}$ & 16.8 & 0.654 & & & \\
\hline \multirow[t]{2}{*}{ Nuts (g/day) } & baseline & 10.7 & 14.2 & 11.2 & 12.6 & 10.0 & 12.7 & 10.0 & 13.6 & 10.9 & 11.5 & 0.904 & $<0.001$ & 0.634 & 0.192 \\
\hline & changes & 3.1 & 16.4 & $5.3 * *$ & 17.1 & $5.5^{* *}$ & 15.8 & $9.5^{* *}$ & 17.3 & $4.2 * *$ & 14.0 & 0.039 & & & \\
\hline \multirow[t]{2}{*}{ Wine (g/day) } & baseline & 104.9 & 144.8 & 97.0 & 138.3 & 103.9 & 171.5 & 98.2 & 136.2 & 81.2 & 125.6 & 0.739 & 0.013 & 0.647 & 0.984 \\
\hline & changes & -8.4 & 124.2 & -13.3 & 129.4 & -17.1 & 111.3 & $-18.1 *$ & 95.3 & -14.5 & 91.9 & 0.971 & & & \\
\hline \multirow[t]{2}{*}{ Tea $(\mathrm{mL})$} & baseline & 4.8 & 14.5 & 4.6 & 15.1 & 6.4 & 17.0 & 5.2 & 12.5 & 7.6 & 21.1 & 0.605 & 0.401 & 0.479 & 0.172 \\
\hline & changes & 0.1 & 16.6 & -1.9 & 14.5 & -1.8 & 16.7 & 3.2 & 24.9 & -2.0 & 22.4 & 0.204 & & & \\
\hline \multirow[t]{2}{*}{ Coffee $(\mathrm{mL})$} & baseline & 39.1 & 58.4 & 36.7 & 52.4 & 30.7 & 43.0 & 35.0 & 47.1 & 30.9 & 43.0 & 0.717 & 0.002 & 0.546 & 0.098 \\
\hline & changes & -11.5 * & 50.1 & -1.8 & 50.2 & $-7.3 *$ & 36.8 & $-13.1^{* *}$ & 47.0 & 3.7 & 51.3 & 0.048 & & & \\
\hline \multirow[t]{2}{*}{ Total carbohydrates (g/day) } & baseline & 237.5 & 43.2 & 242.1 & 38.0 & 237.0 & 41.7 & 235.8 & 36.3 & 239.7 & 34.8 & 0.682 & 0.769 & 0.114 & 0.025 \\
\hline & changes & 4.5 & 78.6 & -10.3 & 71.6 & 5.7 & 72.4 & $-13.2 *$ & 68.1 & -0.4 & 64.6 & 0.166 & & & \\
\hline Protein (g/day) & baseline & 90.3 & 43.2 & 94.9 & 38.0 & 89.7 & 41.7 & 88.5 & 36.3 & 92.5 & 34.8 & 0.682 & 0.274 & 0.307 & 0.474 \\
\hline & changes & 2.3 & 47.6 & -3.0 & 41.4 & 6.1 & 46.9 & 2.5 & 40.9 & 4.6 & 39.6 & 0.592 & & & \\
\hline
\end{tabular}


Table 2. Cont.

\begin{tabular}{|c|c|c|c|c|c|c|c|c|c|c|c|c|c|c|c|}
\hline & & \multicolumn{14}{|c|}{ TPE (mg GAE/g Creatinine) } \\
\hline & & \multirow{2}{*}{\multicolumn{2}{|c|}{$\frac{\mathrm{Q} 1}{(<79.02)}$}} & \multirow{2}{*}{\multicolumn{2}{|c|}{$\frac{\text { Q2 }}{(79.03-99.50)}$}} & \multirow{2}{*}{\multicolumn{2}{|c|}{$\begin{array}{c}\mathrm{Q3} \\
(99.51-124.53)\end{array}$}} & \multirow{2}{*}{\multicolumn{2}{|c|}{$\begin{array}{c}\mathrm{Q} 4 \\
(124.54-160.06)\end{array}$}} & \multirow{2}{*}{\multicolumn{2}{|c|}{$\frac{\text { Q5 }}{(>160.07)}$}} & \multirow{3}{*}{$\begin{array}{c}p^{\mathrm{b}} \\
\text { ANOVA }\end{array}$} & \multirow{2}{*}{\multicolumn{3}{|c|}{$p^{\mathrm{c}}$}} \\
\hline & & & & & & & & & & & & & & & \\
\hline & & Mean & SD & Mean & SD & Mean & SD & Mean & SD & Mean & SD & & TIME & GROUP & INTERACTION \\
\hline \multirow[t]{2}{*}{ Total Fat (g/day) } & baseline & 100.4 & 13.6 & 100.9 & 12.7 & 102.8 & 13.7 & 102.7 & 12.9 & 104.5 & 13.6 & 0.132 & $<0.001$ & 0.235 & 0.981 \\
\hline & changes & $10.0 * *$ & 30.8 & $9.2^{* *}$ & 28.5 & $10.9^{* *}$ & 28.0 & $8.5^{* *}$ & 26.9 & $9.4^{* *}$ & 31.5 & 0.980 & & & \\
\hline \multirow[t]{2}{*}{ Fiber (g/day) } & baseline & 24.2 & 6.0 & 24.5 & 6.5 & 24.2 & 6.5 & 25.8 & 5.8 & 25.5 & 5.6 & 0.256 & 0.006 & 0.632 & 0.013 \\
\hline & changes & 1.1 & 8.2 & 1.4 & 9.7 & $3.2 * *$ & 8.4 & 0.2 & 8.2 & 1.0 & 7.8 & 0.107 & & & \\
\hline \multirow[t]{2}{*}{ Alcohol (g/day) } & baseline & 13.5 & 5.6 & 13.6 & 5.2 & 13.7 & 5.3 & 13.3 & 4.7 & 14.2 & 4.9 & 0.780 & 0.032 & 0.961 & 0.765 \\
\hline & changes & -0.2 & 17.8 & -1.6 & 15.0 & -1.2 & 16.7 & -1.9 & 14.4 & $-3.2 *$ & 14.2 & 0.707 & & & \\
\hline \multirow[t]{2}{*}{ SFA (g/day) } & baseline & 24.9 & 11.2 & 24.2 & 9.7 & 24.5 & 8.3 & 25.7 & 9.1 & 23.9 & 9.9 & 0.663 & 0.541 & 0.772 & 0.266 \\
\hline & changes & -0.5 & 12.9 & -0.1 & 10.6 & 0.7 & 9.8 & -1.5 & 11.1 & 1.6 & 11.3 & 0.301 & & & \\
\hline \multirow[t]{2}{*}{ MUFA (g/day) } & baseline & 52.1 & 16.8 & 51.3 & 15.4 & 52.4 & 16.0 & 53.6 & 14.5 & 52.4 & 15.0 & 0.828 & $<0.001$ & 0.941 & 0.694 \\
\hline & changes & 6.0 ** & 20.3 & $7.5 * *$ & 19.2 & $7.4^{* *}$ & 17.5 & $4.8^{* *}$ & 18.1 & $7.1 * *$ & 19.0 & 0.798 & & & \\
\hline \multirow[t]{2}{*}{ PUFA (g/day) } & baseline & 15.6 & 5.2 & 15.4 & 5.7 & 15.5 & 5.8 & 16.0 & 5.2 & 15.5 & 5.5 & 0.887 & $<0.001$ & 0.575 & 0.670 \\
\hline & changes & $3.0^{* *}$ & 7.7 & $3.1 * *$ & 7.8 & $3.4^{* *}$ & 7.1 & $3.4^{* *}$ & 8.1 & $4.2^{* *}$ & 8.9 & 0.800 & & & \\
\hline \multirow[t]{2}{*}{ Folic acid ( $\mu \mathrm{g} /$ day) } & baseline & 379.3 & 89.5 & 373.3 & 90.5 & 369.8 & 76.5 & 394.4 & 98.8 & 394.2 & 85.7 & 0.152 & $<0.001$ & 0.447 & 0.086 \\
\hline & changes & $42.7^{* *}$ & 91.3 & $46.5^{* *}$ & 99.7 & $65.7^{* *}$ & 92.6 & $39.2^{* *}$ & 91.6 & $38.7^{* *}$ & 100.4 & 0.196 & & & \\
\hline \multirow[t]{2}{*}{ Cholesterol (mg/day) } & baseline & 354.9 & 92.4 & 340.0 & 92.2 & 351.4 & 93.3 & 353.5 & 81.6 & 359.1 & 95.5 & 0.505 & 0.234 & 0.406 & 0.329 \\
\hline & changes & 0.2 & 115.0 & 11.9 & 101.3 & 11.4 & 109.2 & -3.5 & 105.5 & 15.2 & 112.6 & 0.644 & & & \\
\hline \multirow[t]{2}{*}{$\mathrm{Na}$ (mg/day) } & baseline & 2331.2 & 570.5 & 2254.1 & 499.7 & 2272.1 & 480.3 & 2286.1 & 491.3 & 2298.5 & 447.1 & 0.815 & 0.257 & 0.258 & 0.319 \\
\hline & changes & -64.0 & 941.6 & -83.1 & 728.6 & 10.6 & 730.0 & $-184.2^{* *}$ & 713.4 & 10.8 & 752.6 & 0.306 & & & \\
\hline \multirow[t]{2}{*}{$\mathrm{K}$ (mg/day) } & baseline & 4130.5 & 769.6 & 4218.7 & 756.2 & 4208.9 & 696.0 & 4312.6 & 808.1 & 4410.2 & 748.0 & 0.069 & $<0.001$ & 0.246 & 0.067 \\
\hline & changes & $379.8 * *$ & 1070.7 & $286.0^{* *}$ & 1042.2 & $497.3^{* *}$ & 1007.2 & $249.1 *$ & 1116.2 & 268.1 * & 1100.4 & 0.389 & & & \\
\hline \multirow[t]{2}{*}{$\mathrm{Mg}$ (mg/day) } & baseline & 355.0 & 64.0 & 361.1 & 68.6 & 356. & 56.6 & 368.6 & 58.2 & 374.8 & 61.9 & 0.147 & $<0.001$ & 0.474 & 0.056 \\
\hline & changes & $30.7^{* *}$ & 97.9 & $26.8^{* *}$ & 99.2 & $42.9^{* *}$ & 87.9 & $22.0^{* *}$ & 87.3 & 23.9 * & 95.9 & 0.481 & & & \\
\hline \multirow[t]{2}{*}{ P-14 score } & baseline & 8.9 & 1.8 & 9.0 & 1.8 & 9.2 & 1.9 & 8.8 & 1.9 & 9.1 & 1.8 & 0.441 & $<0.001$ & 0.370 & 0.571 \\
\hline & changes & $1.6^{* *}$ & 2.5 & $1.8^{* *}$ & 1.9 & $1.7^{* *}$ & 2.1 & $1.9^{* *}$ & 2.0 & $1.6^{* *}$ & 2.1 & 0.626 & & & \\
\hline \multirow[t]{2}{*}{ Energy intake (Kcal/day) } & baseline & 2508.8 & 582.3 & 2285.5 & 578.5 & 2338.5 & 463.0 & 2262.2 & 510.7 & 2191.7 & 470.7 & $<0.001$ & 0.034 & $<0.001$ & 0.234 \\
\hline & changes & -25.9 & 627.6 & 32.0 & 497.4 & $100.2 *$ & 503.9 & 8.9 & 564.0 & $157.8^{* *}$ & 520.1 & 0.089 & & & \\
\hline
\end{tabular}

TPE: total polyphenol excretion; GAE: gallic acid equivalent; EVOO: Extra Virgin Olive Oil; SFA: saturated fatty acids; MUFA: monounsaturated fatty acids; PUFA: polyunsaturated fatty acids; Na: sodium; K: potassium; Mg: magnesium; $p$-14: 14 item dietary score test to appraise adherence of participants to the Mediterranean diet (it includes questions about consumption of fruits, vegetables, meat, fish, carbonic beverages, legumes, nuts, olive oil, wine, and culinary methods) [28]. ${ }^{a}$ Data are given as means (SD); $p<0.05$ indicates statistical significance. Values with asterisks are statistically different from baseline by paired-samples $t$-test $\left({ }^{*} p<0.05 ;{ }^{* *} p<0.01\right) .{ }^{\mathrm{b}}$ Data were analyzed by one-way ANOVA. ${ }^{\mathrm{c}}$ Data analyzed by repeated-measures two-factor ANOVA. 
The associations between TPE and obesity indexes were analyzed by linear regression models (Table 3). For total participants, significant inverse associations were found between quintiles of TPE at the fifth year and BW $(\beta=-1.004 ; p=0.002)$, BMI $(\beta=-0.320 ; p=0.005)$, WC $(\beta=-0.742 ; p=0.013)$, and WHtR ( $\beta=-0.408 ; p=0.036)$ after adjustment for potential confounders. For males, inverse associations were found in BW ( $\beta=-0.959 ; p=0.039)$ and BMI $(\beta=-0.301 ; p=0.034)$, while, for females, only an inverse association was found in BMI $(\beta=-0.332 ; p=0.046)$ after a full adjustment.

Table 3. Multiple linear regression analyses with obesity indexes and quintiles of TPE at the fifth year for male, female, and total participants.

\begin{tabular}{|c|c|c|c|c|c|c|c|c|}
\hline & & & $\beta$ & SE & Beta & Significance & & \\
\hline \multirow[t]{9}{*}{ BW (kg) } & Male & Model 1 & -1.446 & 0.440 & -0.195 & 0.001 & -2.313 & -0.580 \\
\hline & & Model 2 & -1.259 & 0.440 & -0.170 & 0.005 & -2.126 & -0.392 \\
\hline & & Model 3 & -0.959 & 0.461 & -0.131 & 0.039 & -1.868 & -0.050 \\
\hline & Female & Model 1 & -1.103 & 0.415 & -0.153 & 0.008 & -1.920 & -0.287 \\
\hline & & Model 2 & -0.756 & 0.414 & -0.105 & 0.069 & -1.571 & 0.058 \\
\hline & & Model 3 & -0.757 & 0.431 & -0.107 & 0.080 & -1.606 & 0.091 \\
\hline & Total & Model 1 & -2.350 & 0.331 & -0.285 & $<0.001$ & -3.000 & -1.700 \\
\hline & & Model 2 & -1.070 & 0.315 & -0.130 & 0.001 & -1.689 & -0.451 \\
\hline & & Model 3 & -1.004 & 0.320 & -0.124 & 0.002 & -1.634 & -0.375 \\
\hline \multirow[t]{9}{*}{$\mathrm{BMI}\left(\mathrm{kg} / \mathrm{m}^{2}\right)$} & Male & Model 1 & -0.405 & 0.135 & -0.179 & 0.003 & -0.670 & -0.139 \\
\hline & & Model 2 & -0.370 & 0.136 & -0.164 & 0.007 & -0.639 & -0.102 \\
\hline & & Model 3 & -0.301 & 0.141 & -0.135 & 0.034 & -0.579 & -0.023 \\
\hline & Female & Model 1 & -0.344 & 0.156 & -0.127 & 0.028 & -0.652 & -0.037 \\
\hline & & Model 2 & -0.296 & 0.160 & -0.110 & 0.064 & -0.611 & 0.018 \\
\hline & & Model 3 & -0.332 & 0.165 & -0.123 & 0.046 & -0.657 & -0.007 \\
\hline & Total & Model 1 & -0.295 & 0.104 & -0.118 & 0.005 & -0.499 & -0.090 \\
\hline & & Model 2 & -0.328 & 0.110 & -0.131 & 0.003 & -0.544 & -0.111 \\
\hline & & Model 3 & -0.320 & 0.113 & -0.129 & 0.005 & -0.541 & -0.098 \\
\hline \multirow[t]{9}{*}{$\mathrm{WC}(\mathrm{cm})$} & Male & Model 1 & -0.769 & 0.364 & -0.127 & 0.036 & -1.487 & -0.052 \\
\hline & & Model 2 & -0.786 & 0.369 & -0.130 & 0.034 & -1.513 & -0.059 \\
\hline & & Model 3 & -0.516 & 0.378 & -0.087 & 0.173 & -1.260 & 0.228 \\
\hline & Female & Model 1 & -0.546 & 0.409 & -0.078 & 0.183 & -1.351 & 0.259 \\
\hline & & Model 2 & -0.527 & 0.419 & -0.075 & 0.209 & -1.351 & 0.297 \\
\hline & & Model 3 & -0.701 & 0.434 & -0.101 & 0.108 & -1.556 & 0.154 \\
\hline & Total & Model 1 & -1.500 & 0.296 & -0.208 & $<0.001$ & -2.082 & -0.918 \\
\hline & & Model 2 & -0.721 & 0.293 & -0.100 & 0.014 & -1.296 & -0.147 \\
\hline & & Model 3 & -0.742 & 0.297 & -0.104 & 0.013 & -1.326 & -0.158 \\
\hline \multirow[t]{9}{*}{ WHtR $(\mathrm{cm} / \mathrm{m})$} & Male & Model 1 & -0.340 & 0.220 & -0.093 & 0.124 & -0.773 & 0.094 \\
\hline & & Model 2 & -0.385 & 0.223 & -0.105 & 0.085 & -0.823 & 0.054 \\
\hline & & Model 3 & -0.258 & 0.229 & -0.072 & 0.261 & -0.710 & 0.193 \\
\hline & Female & Model 1 & -0.246 & 0.276 & -0.052 & 0.374 & -0.789 & 0.298 \\
\hline & & Model 2 & -0.332 & 0.282 & -0.070 & 0.240 & -0.887 & 0.223 \\
\hline & & Model3 & -0.501 & 0.294 & -0.106 & 0.089 & -1.079 & 0.078 \\
\hline & Total & Model 1 & -0.298 & 0.178 & -0.070 & 0.094 & -0.648 & 0.051 \\
\hline & & Model 2 & -0.367 & 0.189 & -0.087 & 0.052 & -0.739 & 0.004 \\
\hline & & Model 3 & -0.408 & 0.194 & -0.097 & 0.036 & -0.788 & -0.028 \\
\hline
\end{tabular}

BW: body weight; BMI: body mass index; WC: waist circumference; WHtR: waist-to-height ratio. TPE: total polyphenol excretion (mg GAE/g creatinine). $\beta$ : Non-standardized coefficient (regression line coefficient); SE: Standard error; Beta: Standardized coefficient; CI: Confidence interval; $p$ : two-sided test of significance. Model 1, unadjusted; Model 2 was adjusted for sex (only for total participants), age, and intervention groups; Model 3 adjusted as in Model 2 plus smoking status (never, current, former), family history of CHD, physical activity, hypertension, diabetes, dyslipidemia, marital status (single, married, divorced, widowed), education level (primary school, high school, university), medication used (antihypertensive drugs, vitamins, insulin, oral hypoglycemic drugs, aspirin, or other antiplatelet drug supplements taken in the last month), recruitment centers, 14 unit Mediterranean diet score, and energy intake at baseline.

Table 4 shows the odds ratio (OR) and a 95\% CI for obesity according to the quintile of TPE at the fifth year. In fully adjusted models, for total participants in the category of highest TPE had a lower prevalence of obesity $(\mathrm{OR}=0.346,95 \% \mathrm{CI}=0.176$ to 0.178 ; $p$-trend $=0.039)$ than those in the 
lowest category. For males, compared with the reference group, the top quintile group (Q5) showed significant reduction in the prevalence of obesity $(\mathrm{OR}=0.340,95 \% \mathrm{CI}=0.146$ to 0.792 in Model 2).

Table 4. Multivariate adjusted odds ratios (95\% confidence interval (CI)) for prevalent obesity (213 cases) after a five years follow up.

\begin{tabular}{|c|c|c|c|c|c|c|c|c|c|c|c|c|c|c|c|}
\hline \multirow{4}{*}{$\begin{array}{c}\text { Male } \\
\text { (90 case) }\end{array}$} & \multirow{4}{*}{$\begin{array}{l}\text { Model } 1 \\
\text { Model } 2 \\
\text { Model } 3\end{array}$} & \multirow{4}{*}{$\begin{array}{c}\text { Q1 } \\
1 \text { (ref.) } \\
1 \text { (ref.) } \\
1 \text { (ref.) }\end{array}$} & \multirow{4}{*}{$\begin{array}{c}\text { Q2 } \\
0.531 \\
0.559 \\
0.586\end{array}$} & \multicolumn{2}{|c|}{$95 \% \mathrm{CI}$} & \multirow{2}{*}{$\frac{\text { Q3 }}{0.545}$} & \multicolumn{2}{|c|}{$95 \%$ CI } & \multirow{2}{*}{$\begin{array}{l}\mathrm{Q4} \\
0.488\end{array}$} & \multicolumn{2}{|c|}{$95 \%$ CI } & \multirow{2}{*}{$\frac{\text { Q5 }}{0.313}$} & \multicolumn{2}{|c|}{$95 \% \mathrm{CI}$} & \multirow{2}{*}{$\begin{array}{c}p \\
0.095\end{array}$} \\
\hline & & & & 0.244 & 1.155 & & 0.250 & 1.188 & & 0.223 & 1.070 & & 0.135 & 0.722 & \\
\hline & & & & 0.254 & 1.229 & 0.571 & 0.260 & 1.254 & 0.520 & 0.235 & 1.148 & 0.340 & 0.146 & 0.792 & 0.159 \\
\hline & & & & 0.243 & 1.416 & 0.588 & 0.238 & 1.452 & 0.511 & 0.204 & 1.283 & 0.387 & 0.146 & 1.029 & 0.418 \\
\hline \multirow{3}{*}{$\begin{array}{l}\text { Female } \\
\text { (123 case) }\end{array}$} & Model 1 & 1 (ref.) & 0.934 & 0.454 & 1.924 & 0.791 & 0.384 & 1.628 & 0.643 & 0.310 & 1.333 & 0.429 & 0.200 & 0.919 & 0.195 \\
\hline & Model 2 & 1 (ref.) & 1.041 & 0.497 & 2.182 & 0.844 & 0.403 & 1.768 & 0.769 & 0.361 & 1.638 & 0.493 & 0.226 & 1.078 & 0.352 \\
\hline & Model 3 & 1 (ref.) & 1.257 & 0.538 & 2.934 & 0.748 & 0.317 & 1.764 & 0.595 & 0.244 & 1.450 & 0.461 & 0.181 & 1.170 & 0.223 \\
\hline \multirow{3}{*}{$\begin{array}{c}\text { Total } \\
\text { (213 case) }\end{array}$} & Model 1 & 1 (ref.) & 0.639 & 0.375 & 1.089 & 0.769 & 0.454 & 1.302 & 0.664 & 0.390 & 1.129 & 0.450 & 0.259 & 0.782 & 0.073 \\
\hline & Model 2 & 1 (ref.) & 0.597 & 0.344 & 1.035 & 0.691 & 0.400 & 1.192 & 0.618 & 0.350 & 1.091 & 0.383 & 0.211 & 0.694 & 0.036 \\
\hline & Model 3 & 1 (ref.) & 0.604 & 0.332 & 1.100 & 0.720 & 0.399 & 1.300 & 0.560 & 0.298 & 1.054 & 0.346 & 0.176 & 0.678 & 0.039 \\
\hline
\end{tabular}

Quintiles for males: Q1 < 70.61; Q2: 70.62-88.94; Q3: 88.95-108.61; Q4: 108.62-137.11; Q5 > 137.11; Quintiles for females: Q1 < 91.67; Q2: 91.68-113.96; Q3: 113.97-138.28; Q4: 138.29-181.01; Q5 > 181.01; Quintiles for total: Q1 < 79.02; Q2: 79.03-99.50; Q3: 99.51-124.53; Q4: 124.54-160.06; Q5 > 160.06. TPE is expressed as mg GAE/g creatinine. Obesity was defined as BMI $>30 \mathrm{~kg} / \mathrm{m}^{2}$. Model 1, unadjusted; Model 2 was adjusted for sex (only for total participants), age, and intervention groups; Model 3 adjusted as in Model 2 plus smoking status (never, current, former), family history of CHD, physical activity, hypertension, diabetes, dyslipidemia, marital status (single, married, divorced, widowed), education level (primary school, high school, university), medication used (antihypertensive drugs, vitamins, insulin, oral hypoglycemic drugs, aspirin or other antiplatelet drug supplements taken in the last month) recruitment centers, 14 unit Mediterranean diet score, and energy intake at baseline.

Table 5 shows the incidence of obesity after five years of intervention, conducted in subjects without obesity at baseline and adjusted for TPE and BW at baseline and other co-variables. The results show significant reduction in the incidence of obesity (odds ratio $(\mathrm{OR})=0.095,95 \%$ confidence interval (CI) 0.018 to 0.498 ; $p$-trend, 0.018 ) at the end of the follow up after adjustments.

Table 5. Association between TPE after five years of follow up and the incidence of obesity (39 new-onset case).

\begin{tabular}{lcccccccccccccc}
\hline & Q1 & Q2 & \multicolumn{2}{c}{$\mathbf{9 5 \%}$ CI } & Q3 & 95\% CI & Q4 & \multicolumn{2}{c}{ 95\% CI } & Q5 & 95\% CI & $p$ \\
\hline Model 1 & 1 (ref.) & 0.912 & $(0.337$ & $2.468)$ & 0.676 & $(0.253$ & $1.810)$ & 0.351 & $(0.142$ & $0.866)$ & 0.406 & $(0.164$ & $1.005)$ & 0.054 \\
Model 2 & 1 (ref.) & 0.454 & $(0.185$ & $1.115)$ & 0.235 & $(0.072$ & $0.767)$ & 0.285 & $(0.093$ & $0.868)$ & 0.145 & $(0.037$ & $0.558)$ & 0.014 \\
Model 3 & 1 (ref.) & 0.382 & $(0.146$ & $1.001)$ & 0.193 & $(0.055$ & $0.676)$ & 0.272 & $(0.084$ & $0.885)$ & 0.119 & $(0.028$ & $0.505)$ & 0.014 \\
Model 4 & 1 (ref.) & 0.366 & $(0.126$ & $1.062)$ & 0.156 & $(0.040$ & $0.612)$ & 0.218 & $(0.054$ & $0.881)$ & 0.095 & $(0.018$ & $0.498)$ & 0.018 \\
\hline
\end{tabular}

Model 1, unadjusted; Model 2 was adjusted for baseline TPE and baseline BW; Model 3 was adjusted as in Model 2 plus sex, age, and intervention groups; Model 4 was adjusted as in Model 3 plus smoking status (never, current, former), family history of CHD, physical activity, hypertension, diabetes, dyslipidemia, marital status (single, married, divorced, widowed), education level (primary school, high school, university), medication used (antihypertensive drugs, vitamins, insulin, oral hypoglycemic drugs, aspirin, or other antiplatelet drug supplements taken in the last month) recruitment centers, 14 unit Mediterranean diet score, and energy intake at baseline.

Table 6 shows the associations between TPE at the fifth year and changes in anthropometric parameters, analyzed by linear regression models. For total participants, inverse associations were found between changes in BW $(\beta=-0.363 ; p=0.024)$ and BMI $(\beta=-0.145 ; p=0.023)$ and TPE in the fifth year after adjustment. For males, there was not any inverse association, while for females, inverse associations were found for changes in BW $(\beta=-0.568 ; p=0.008)$ and BMI $(\beta=-0.221 ; p=0.017)$ after adjustment. 
Table 6. Multiple linear regression analyses with changes in anthropometric parameters and quintiles of TPE at the fifth year.

\begin{tabular}{|c|c|c|c|c|c|c|c|c|}
\hline & & & $\beta$ & SE & Beta & $p$ & \multicolumn{2}{|c|}{$95 \%$ CI } \\
\hline \multirow{12}{*}{$\begin{array}{l}\text { Changes in BW } \\
(\mathrm{kg})\end{array}$} & Male & Model 1 & -0.098 & 0.211 & -0.028 & 0.642 & -0.514 & 0.318 \\
\hline & & Model 2 & -0.229 & 0.217 & -0.066 & 0.294 & -0.657 & 0.199 \\
\hline & & Model 3 & -0.186 & 0.217 & -0.053 & 0.393 & -0.614 & 0.242 \\
\hline & & Model 4 & -0.037 & 0.233 & -0.011 & 0.872 & -0.495 & 0.421 \\
\hline & Female & Model 1 & -0.648 & 0.193 & -0.193 & 0.001 & -1.027 & -0.269 \\
\hline & & Model 2 & -0.664 & 0.196 & -0.197 & 0.001 & -1.049 & -0.279 \\
\hline & & Model 3 & -0.573 & 0.197 & -0.17 & 0.004 & -0.961 & -0.185 \\
\hline & & Model 4 & -0.568 & 0.213 & -0.169 & 0.008 & -0.987 & -0.149 \\
\hline & Total & Model 1 & -0.429 & 0.142 & -0.125 & 0.003 & -0.709 & -0.149 \\
\hline & & Model 2 & -0.539 & 0.15 & -0.157 & $<0.001$ & -0.835 & -0.244 \\
\hline & & Model 3 & -0.436 & 0.153 & -0.127 & 0.005 & -0.737 & -0.135 \\
\hline & & Model 4 & -0.363 & 0.161 & -0.108 & 0.024 & -0.68 & -0.047 \\
\hline \multirow{12}{*}{$\begin{array}{l}\text { Changes in BMI } \\
\left(\mathrm{kg} / \mathrm{m}^{2}\right)\end{array}$} & Male & Model 1 & -0.04 & 0.075 & -0.033 & 0.589 & -0.187 & 0.107 \\
\hline & & Model 2 & -0.087 & 0.077 & -0.07 & 0.259 & -0.238 & 0.064 \\
\hline & & Model 3 & -0.072 & 0.077 & -0.059 & 0.348 & -0.224 & 0.079 \\
\hline & & Model 4 & -0.019 & 0.082 & -0.016 & 0.817 & -0.18 & 0.142 \\
\hline & Female & Model 1 & -0.256 & 0.083 & -0.177 & 0.002 & -0.419 & -0.092 \\
\hline & & Model 2 & -0.262 & 0.084 & -0.181 & 0.002 & -0.428 & -0.096 \\
\hline & & Model 3 & -0.223 & 0.085 & -0.154 & 0.009 & -0.391 & -0.056 \\
\hline & & Model 4 & -0.221 & 0.092 & -0.153 & 0.017 & -0.402 & -0.04 \\
\hline & Total & Model 1 & -0.176 & 0.056 & -0.13 & 0.002 & -0.286 & -0.065 \\
\hline & & Model 2 & -0.215 & 0.059 & -0.159 & $<0.001$ & -0.331 & -0.098 \\
\hline & & Model 3 & -0.172 & 0.06 & -0.128 & 0.005 & -0.291 & -0.054 \\
\hline & & Model 4 & -0.145 & 0.064 & -0.109 & 0.023 & -0.27 & -0.02 \\
\hline \multirow{12}{*}{$\begin{array}{l}\text { Changes in WC } \\
(\mathrm{cm})\end{array}$} & Male & Model 1 & -0.066 & 0.233 & -0.017 & 0.776 & -0.525 & 0.392 \\
\hline & & Model 2 & -0.209 & 0.241 & -0.054 & 0.386 & -0.684 & 0.265 \\
\hline & & Model 3 & -0.17 & 0.242 & -0.044 & 0.484 & -0.647 & 0.307 \\
\hline & & Model 4 & -0.108 & 0.249 & -0.029 & 0.666 & -0.599 & 0.383 \\
\hline & Female & Model 1 & -0.34 & 0.275 & -0.072 & 0.217 & -0.88 & 0.2 \\
\hline & & Model 2 & -0.325 & 0.28 & -0.069 & 0.247 & -0.877 & 0.227 \\
\hline & & Model 3 & -0.31 & 0.286 & -0.066 & 0.279 & -0.872 & 0.253 \\
\hline & & Model 4 & -0.42 & 0.311 & -0.089 & 0.178 & -1.033 & 0.192 \\
\hline & Total & Model 1 & -0.237 & 0.181 & -0.055 & 0.190 & -0.592 & 0.118 \\
\hline & & Model 2 & -0.302 & 0.192 & -0.070 & 0.118 & -0.680 & 0.076 \\
\hline & & Model 3 & -0.252 & 0.198 & -0.059 & 0.203 & -0.640 & 0.136 \\
\hline & & Model 4 & -0.269 & 0.207 & -0.063 & 0.195 & -0.676 & 0.138 \\
\hline \multirow{12}{*}{$\begin{array}{l}\text { Changes in WHtR } \\
(\mathrm{cm} / \mathrm{m})\end{array}$} & Male & Model 1 & -0.066 & 0.233 & -0.017 & 0.776 & -0.525 & 0.392 \\
\hline & & Model 2 & -0.209 & 0.241 & -0.054 & 0.386 & -0.684 & 0.265 \\
\hline & & Model 3 & -0.17 & 0.242 & -0.044 & 0.484 & -0.647 & 0.307 \\
\hline & & Model 4 & -0.108 & 0.249 & -0.029 & 0.666 & -0.599 & 0.383 \\
\hline & Female & Model 1 & -0.202 & 0.179 & -0.066 & 0.262 & -0.555 & 0.152 \\
\hline & & Model 2 & -0.194 & 0.183 & -0.063 & 0.29 & -0.555 & 0.166 \\
\hline & & Model 3 & -0.184 & 0.187 & -0.06 & 0.325 & -0.552 & 0.183 \\
\hline & & Model 4 & -0.262 & 0.204 & -0.085 & 0.199 & -0.663 & 0.139 \\
\hline & Total & Model 1 & -0.142 & 0.114 & -0.052 & 0.216 & -0.367 & 0.083 \\
\hline & & Model 2 & -0.184 & 0.122 & -0.068 & 0.131 & -0.424 & 0.055 \\
\hline & & Model 3 & -0.155 & 0.125 & -0.057 & 0.215 & -0.401 & 0.091 \\
\hline & & Model 4 & -0.167 & 0.131 & -0.062 & 0.203 & -0.426 & 0.091 \\
\hline
\end{tabular}

TPE: total polyphenol excretion; GAE: gallic acid equivalent; BW: body weight; BMI: body mass index. WC: waist circumference; WHtR: waist-to-height ratio. Model 1, unadjusted; Model 2 was adjusted for baseline TPE and baseline BW; Model 3 was adjusted as in Model 2 plus sex (only for total participants), age, and intervention groups; Model 4 was adjusted as in Model 3 plus smoking status (never, current, former), family history of CHD, physical activity, hypertension, diabetes, dyslipidemia, marital status (single, married, divorced, widowed), education level (primary school, high school, university), medication used (antihypertensive drugs, vitamins, insulin, oral hypoglycemic drugs, aspirin, or other antiplatelet drug supplements taken in the last month) recruitment centers, 14 unit Mediterranean diet score, and energy intake at baseline.

Changes in obesity parameters for male, female, and total participants between baseline and end of follow up were observed (Table S1). For total participants, subjects in the highest TPE category had the lowest BW $(70.29 \pm 10.25 \mathrm{~kg})$ and BMI $\left(28.40 \pm 3.75 \mathrm{~kg} / \mathrm{m}^{2}\right)$ after the intervention. Inversely, 
those participants in the first quintile of TPE had significantly higher WC $(101.41 \pm 9.35 \mathrm{~cm})$ and WHtR $(61.80 \pm 5.15)$ compared with baseline values. For males, there was a significant inverse trend among quintiles and BW and BMI both at baseline and the fifth year. Also a significant reduction was observed comparing the top quintile with the bottom quintile both at baseline and the fifth year. For females, there was a significant reduction of BW and BMI in the top quintile groups after five years of intervention.

Table S2 shows the associations between changes in anthropometric parameters and changes in TPE over five years with linear regression models. For total participants, inverse associations were found between changes in BW ( $\beta=-0.355 ; p=0.036)$, BMI ( $\beta=-0.139 ; p=0.037)$ and TPE five years after adjustment. For males, there was not any inverse association, while, for females, inverse associations were found for changes in BW ( $\beta=-0.723 ; p=0.003)$, BMI $(\beta=-0.283 ; p=0.006)$, and WC $(\beta=-0.701 ; p=0.046)$ after adjustment.

\section{Discussion}

In this five years study conducted in elderly participants at high cardiovascular risk, a higher total polyphenol intake, expressed as TPE, was inversely associated with weight parameters including BW, BMI, WC, and WHtR, as well as with the prevalence of obesity after a five years follow up, suggesting that polyphenols could be considered an independent contributor to the weight loss effects of a Mediterranean diet.

Several PREDIMED sub-trials have reported a range of mechanisms for the weight loss effects of a Mediterranean diet, including a high ingestion of dietary fiber, antioxidants, unsaturated fatty acids, extra virgin olive oil, nuts, and moderate wine consumption [29-35]. The reduction we observed in weight parameters might be partly attributed to the intake of the aforementioned food items; however, in the fully adjusted models, we removed their effects by adjusting for adherence to the Mediterranean diet (14 unit MedDiet questionnaire). Furthermore, even though the intake of these foods increased after five years of follow up, none of them showed significant differences within quintile categories at the end of the intervention; therefore, polyphenol intake could be considered an independent factor.

The present findings are consistent with previous reports on the inverse associations between polyphenol intake and weight parameters. A 16 years longitudinal study from the Netherlands associated a higher intake of total flavonols/flavones and catechins with a lower increase in BMI [36]. Other supporting evidence showed a significant decrease of $1.9 \mathrm{~cm}$ in WC and $1.2 \mathrm{~kg}$ in BW after supplementation of catechin-rich green tea for 90 days, although at a much higher dose than habitual intakes [37]. Two 12 week intervention studies also demonstrated the anti-obesity effects of green tea intake, finding a considerable reduction in BW, BMI, WC, and total abdominal fat area [38,39]. Another clinical trial indicated that consumption of normal or high-polyphenolic orange juice reduced body weight in obese or overweight adults, demonstrating an inverse association between polyphenol intake and body weight [10]. On the contrary, the effect of daily decaffeinated green tea intake on weight and body composition were tested among a group population in overweight breast cancer survivors. Results showed a slight but not significant increasing in weight loss after the intervention [40]. Compared with Asian populations, Caucasians show inconsistent results: a study showed no effects on body weight with long-term green tea extract supplementation [11]; another study with relapsing-remitting multiple sclerosis patients using one of the main green tea polyphenols, (-)epigal-locatechin-3-gallate, after three months of consumption showed greater muscle metabolism improvement in males than females [41]; supplementation with resveratrol exerted significant effects on energy metabolism in obese subjects, while another two findings showed ineffectiveness in nonobese women and obese men [42-44]. The results are inconsistent probably because of the different doses of polyphenol intakes, sex-specific effects, sample sizes, or length of duration.

The Mediterranean diet could be considered rich in polyphenol content because it is characterized by a high consumption of fruit and vegetables, virgin olive oil, legumes, and nuts and a moderate consumption of wine [45]. Results from a meta-analysis of 16 randomized controlled trials with a 
Mediterranean diet showed an average reduction in participant weight of $1.75 \mathrm{~kg}$ and a reduction in BMI of $0.57 \mathrm{~kg} / \mathrm{m}^{2}$, as well as a greater reduction in BW of $3.88 \mathrm{~kg}$ under conditions of energy restriction, suggesting that adherence to a Mediterranean diet helps to control weight [46]. The PREDIMED, the European Prospective Investigation into Cancer and Nutrition (EPIC-Spain) cohort and the Seguimiento Universidad de Navarra (SUN) cohort, also in Spain, have shown in the long-term a significantly lower risk of overweightness/obesity associated with better Mediterranean diet adherence [34,47,48]. We observed a $1.22 \mathrm{~kg}$ decrease in BW and 0.50 in BMI in the highest TPE quintile, which partly agrees with previous studies reporting a similar reduction in body weight parameters.

Indexes of abdominal obesity, namely WC and WHtR, were significantly lower in the highest TPE quintile. These parameters are more accurate discriminators of cardiovascular risk than BMI due to the closer relationship between cardiovascular disease and abdominal obesity [49]. In agreement with our findings, in a PREDIMED study, and several other studies, the Mediterranean diet was negatively associated with WC and WHtR [50-52]. Additionally, two feeding trials with green tea polyphenol extracts also showed beneficial effects on abdominal obesity parameters [39,53].

Our results indicate the weight loss effect of polyphenols is higher in females than males. There are several possible reasons to explain the observed results: first, the prevalence of obesity is higher in women than men, and weight loss tends to be lower in obese individuals [54]. In the current study, at baseline, the prevalence of obesity was $32.9 \%$ for males and $44.9 \%$ for females, which is in line with another elderly population in United States [55]. Second, a higher TPE increment was observed when comparing females and males after five years, which may potentially explain the difference in weight loss effectiveness. Third, self-characteristics concerning males and females may also contribute to the difference. Women tend to have higher concentrations of leptin, an appetite regulation hormone that helps to reduce energy intake [56]. Evidence shows a significant inverse association between polyphenol intakes and plasma leptin levels, indicating higher polyphenol intake responses to better weight loss effectiveness [10]. Furthermore, individual differences in the composition of the gut microbiota may also contribute to differences in bioavailability and polyphenolic metabolites, further influencing the weight loss effectiveness [57].

We also found less gain in WC in males, after five years of intervention. The observation could be primarily explained by the greater percentage of muscle mass and mineral mass in males compared with females. Waist circumference is affected by age, body weight, body composition, and fat distribution [58]. Various types of polyphenol help to reduce visceral fat [9]. Additionally, since waist circumference increases with age, it is worth noting that the average age is older in females in our population, which could be considered another possible explanation.

Potential explanations of the observed inverse association between polyphenol intake and weight loss likely involve several mechanisms due to the diversity of polyphenol chemical structures, complex metabolic pathways, and oral bioavailability. Excess adipose mass and adipose tissue expansion results from adipocyte hypertrophy and hyperplasia [59]. Common plausible mechanisms include the suppression of fat absorption and anabolic pathways; inhibition of adipogenesis and lipogenesis; stimulation of catabolic pathways with increment of lipolysis, apoptosis of mature adipocytes, and acid $\beta$-oxidation; reduction of chronic inflammatory response relative to adiposity; and increment in energy expenditure through up-regulating uncoupling protein (UCP1-3) [8,9]. However, knowledge of the anti-obesity effects of polyphenols is limited and only a few specific compounds have been analyzed in this context. For instance, it has been demonstrated that resveratrol, widely present in red grapes and red wine, exerts an anti-obesity action by reducing adipogenesis and increasing apoptosis in mature adipocytes and inhibiting fat accumulation processes and stimulating lipolytic and oxidative pathways in vivo studies and clinical trials $[43,60,61]$. Contrary results have been observed with anthocyanins, water-soluble plant pigments in blue, purple, and red fruits. On one hand, they seem to significantly reduce body weight. This effect may be due to the suppression of lipid synthesis, the up-regulation of adiponectin, which enhances insulin sensitivity, and the reduction in of serum triglycerides and leptin levels $[8,62]$. However, two clinical trials showed non-significant reduction trends in body weight after 
supplementation with food rich in anthocyanins $[63,64]$. The anti-obesity effects of flavonoids, which are a large group of polyphenols found in a wide range of Mediterranean diet foods [65,66], have been mainly attributed to improvement in adipocyte functionality and fat oxidation [67]. Also playing a key role in weight control is the down-regulation of a variety of pro-inflammatory adipocytokines, particularly tumor necrosis factor alpha (TNF- $\alpha$ ) [68]. A clinical trial indicates that the inhibition of intestinal fat absorption may contribute to the weight loss after an ingestion of a green tea beverage enriched with catechins [69]. In summary, even though the intake of some specific polyphenols has been associated with body weight management, there is still not enough evidence for the effect of total polyphenols or some classes of polyphenols, and further studies are needed to explore the mechanisms involved as well as potential synergistic effects among them.

The association between weight loss and improvement in cardiovascular risk factors has been widely discussed. Numerous studies indicate that polyphenol intake reduces cardiovascular risk factors [70,71]. A previous study found a significant improvement in cardiovascular risk factors, with a $5-10 \%$ of weight loss after one year [72]. We also found protective effects on cardiovascular risk factors, including diastolic blood pressure, glucose concentration, and triglycerides concentration, with the same population after five years of intervention [73]. A randomized study conducted in healthy participants, feed with apple polyphenol, also supported that an improvement in cardiovascular risk factor helps to regulate fat metabolism [74].

Some limitations of this study should be noted. First, given that the study was conducted among elderly subjects at high cardiovascular risk, the results cannot be extrapolated to the general population. Second, even though we adjusted for major potential confounders, we still cannot exclude residual confounding from measurements. Third, even though WC and WHtR may reflect abdominal obesity more accurately, they cannot differentiate between fat distribution in visceral adipose tissue and subcutaneous abdominal adipose tissue; hence we cannot conclude if a reduction in abdominal obesity parameters is beneficial to visceral or subcutaneous fat mass or both [75]. Another limitation is the lack of specific measurements of polyphenol metabolism in vivo.

The present study also has several strengths. Its main strong point is the use of TPE, a biomarker of polyphenol intake, which could provide more precise data than measuring total polyphenol intake through self-reported information in FFQs or databases. Another strength is its prospective design. Only a few studies have analyzed the association between total polyphenol intake and weight control, and the current work is the first to associate anti-obesity effects with total polyphenol intake in individuals at high cardiovascular risk $[8,76]$. In addition, the long-term duration of the intervention provides more robust results compared with other short-term trials.

\section{Conclusions}

In summary, with five years of follow up, the present study shows that polyphenol levels expressed as TPE in urine were inversely associated with BW, BMI, WC, and WHtR in an elderly population at high cardiovascular risk. Therefore, we confirmed that a long-term polyphenol-rich diet contributes to body weight loss, which can offer protection from several chronic diseases. For future research, similar studies should be conducted in the general population, and specific mechanisms need to be explored by further clinical trials.

Supplementary Materials: The following are available online at www.mdpi.com/2072-6643/9/5/452/s1, Table S1: Comparisons of obesity indexes, Table S2: Multiple linear regression analyses with changes in anthropometric parameters and changes in quintiles of TPE over five years, File S1: STROBE checklist, File S2: Protocol of the PREDIMED study, File S3: Database of participants.

Acknowledgments: We thank all the participants of the PREDIMED study. This work was supported in part by CICYT (AGL2016-79113-R), the Instituto de Salud Carlos III, ISCIII (CIBEROBN) from the Spanish Ministry of Economy and Competivity (MEC), and Generalitat de Catalunya (GC) 2014 SGR 773. X.G. received support from China Scholarship Council (CSC). Alexander Medina-Remón thanks the 'Juan de la Cierva' postdoctoral program (JCI-2012-13463) from MEC. 
Author Contributions: R.M.L.-R., A.T.-R. and R.E. conceived and designed the experiments; A.T.-R., A.M.-R. and X.G. performed the experiments; X.G., A.T.-R. and A.M.-R. analyzed the data; A.T.-R., R.E., D.C., M.A.M.-G. and M.F. contributed reagents/materials/analysis tools; and X.P.-S., A.T.-R. and R.M.L.-R. wrote the paper. All authors agreed with the manuscript's results and conclusions.

Conflicts of Interest: The authors declare no conflict of interest. The founding sponsors had no role in the design of the study; in the collection, analyses, or interpretation of data; in the writing of the manuscript; or in the decision to publish the results.

\section{References}

1. Ng, M.; Fleming, T.; Robinson, M.; Thomson, B.; Graetz, N.; Margono, C.; Mullany, E.C.; Biryukov, S.; Alfonso, R.; Ali, M.K.; et al. Global, regional, and national prevalence of overweight and obesity in children and adults during 1980-2013: A systematic analysis for the Global Burden of Disease Study 2013. Lancet 2014, 384, 766-781. [CrossRef]

2. World Health Organization. Obesity and Overweight. Available online: http://www.who.int/mediacentre/ factsheets/fs311/en (accessed on 2 August 2016).

3. Directorate-General for Health and Consumers. Strategy for Europe on Nutrition, Overweight and Obesity Related Health Issues; Implementation Progress Report; European Commission: Brussels, Belgium, December 2010.

4. Fitch, A.; Everling, L.; Fox, C.; Goldberg, J.; Heim, C.; Johnson, K.; Kaufman, T.; Kennedy, E.; Kestenbaun, C.; Leslie, D.; et al. Prevention and Management of Obesity for Adults. ICSI Health Care Guideline, 2013. Available online: https://www.healthpartners.com/ucm/groups/public/@hp/@public/documents/ documents/cntrb_037112.pdf (accessed on 2 August 2016).

5. Malik, V.S.; Willett, W.C.; Hu, F.B. Global obesity: Trends, risk factors and policy implications. Nat. Rev. Endocrinol. 2013, 9, 13-27. [CrossRef] [PubMed]

6. Ross, R.; Blair, S.; de Lannoy, L.; Després, J.-P.; Lavie, C.J. Changing the endpoints for determining effective obesity management. Prog. Cardiovasc. Dis. 2015, 57, 330-336. [CrossRef] [PubMed]

7. Scalbert, A.; Williamson, G. Dietary intake and bioavailability of polyphenols. J. Nutr. 2000, 130, $2073-2085$.

8. Meydani, M.; Hasan, S.T. Dietary polyphenols and obesity. Nutrients 2010, 2, 737-751. [CrossRef] [PubMed]

9. Wang, S.; Moustaid-Moussa, N.; Chen, L.; Mo, H.; Shastri, A.; Su, R.; Bapat, P.; Kwun, I.; Shen, C.L. Novel insights of dietary polyphenols and obesity. J. Nutr. Biochem. 2014, 25, 1-18. [CrossRef] [PubMed]

10. Rangel-Huerta, O.D.; Aguilera, C.M.; Martin, M.V.; Soto, M.J.; Rico, M.C.; Vallejo, F.; Tomas-Barberan, F.; Perez-de-la-Cruz, A.J.; Gil, A.; Mesa, M.D. Normal or high polyphenol concentration in orange juice affects antioxidant activity, blood pressure, and body weight in obese or overweight adults. J. Nutr. 2015, 145, 1808-1816. [CrossRef] [PubMed]

11. Janssens, P.L.H.R.; Hursel, R.; Westerterp-Plantenga, M.S. Long-term green tea extract supplementation does not affect fat absorption, resting energy expenditure, and body composition in adults. J. Nutr. 2015, 145, 864-870. [CrossRef] [PubMed]

12. Bell, Z.W.; Canale, R.E.; Bloomer, R.J. A dual investigation of the effect of dietary supplementation with licorice flavonoid oil on anthropometric and biochemical markers of health and adiposity. Lipids Health Dis. 2011, 10, 29. [CrossRef] [PubMed]

13. Dallas, C.; Gerbi, A.; Elbez, Y.; Caillard, P.; Zamaria, N.; Cloarec, M. Clinical study to assess the efficacy and safety of a citrus polyphenolic extract of red orange, grapefruit, and orange (Sinetrol-XPur) on weight management and metabolic parameters in healthy overweight individuals. Phytother. Res. 2014, 28, 212-218. [CrossRef] [PubMed]

14. Most, J.; Goossens, G.H.; Jocken, J.W.E.; Blaak, E.E. Short-term supplementation with a specific combination of dietary polyphenols increases energy expenditure and alters substrate metabolism in overweight subjects. Int. J. Obes. 2014, 38, 698-706. [CrossRef] [PubMed]

15. Barth, S.W.; Koch, T.C.L.; Watzl, B.; Dietrich, H.; Will, F.; Bub, A. Moderate effects of apple juice consumption on obesity-related markers in obese men: Impact of diet-gene interaction on body fat content. Eur. J. Nutr. 2012, 51, 841-850. [CrossRef] [PubMed]

16. Cases, J.; Romain, C.; Dallas, C.; Gerbi, A.; Cloarec, M. Regular consumption of Fiit-ns, a polyphenol extract from fruit and vegetables frequently consumed within the Mediterranean diet, improves metabolic ageing of obese volunteers: A randomized, double-blind, parallel trial. Int. J. Food Sci. Nutr. 2015, 66, 120-125. [CrossRef] [PubMed] 
17. Almoosawi, S.; Fyfe, L.; Ho, C.; Al-Dujaili, E. The effect of polyphenol-rich dark chocolate on fasting capillary whole blood glucose, total cholesterol, blood pressure and glucocorticoids in healthy overweight and obese subjects. Br. J. Nutr. 2010, 103, 842-850. [CrossRef] [PubMed]

18. Medina-Remón, A.; Tresserra-Rimbau, A.; Pons, A.; Tur, J.A.; Martorell, M.; Ros, E.; Buil-Cosiales, P.; Sacanella, E.; Covas, M.I.; Corella, D.; et al. Effects of total dietary polyphenols on plasma nitric oxide and blood pressure in a high cardiovascular risk cohort. The PREDIMED randomized trial. Nutr. Metab. Cardiovasc. Dis. 2015, 25, 60-67. [CrossRef] [PubMed]

19. Medina-Remón, A.; Barrionuevo-González, A.; Zamora-Ros, R.; Andres-Lacueva, C.; Estruch, R.; Martínez-González, M.A.; Diez-Espino, J.; Lamuela-Raventos, R.M. Rapid Folin-Ciocalteu method using microtiter 96-well plate cartridges for solid phase extraction to assess urinary total phenolic compounds, as a biomarker of total polyphenols intake. Anal. Chim. Acta 2009, 634, 54-60. [CrossRef] [PubMed]

20. Zamora-Ros, R.; Rabassa, M.; Cherubini, A.; Urpi-Sarda, M.; Llorach, R.; Bandinelli, S.; Ferrucci, L.; Andres-Lacueva, C. Comparison of 24-h volume and creatinine-corrected total urinary polyphenol as a biomarker of total dietary polyphenols in the Invecchiare InCHIANTI study. Anal. Chim. Acta 2011, 704, 110-115. [CrossRef] [PubMed]

21. Urpi-Sarda, M.; Andres-Lacueva, C.; Rabassa, M.; Ruggiero, C.; Zamora-Ros, R.; Bandinelli, S.; Ferrucci, L.; Cherubini, A. The relationship between urinary total polyphenols and the frailty phenotype in a community-dwelling older population: The InCHIANTI study. J. Gerontol. A Biol. Sci. Med. Sci. 2015, 70, 1141-1147. [CrossRef] [PubMed]

22. Martínez-González, M.Á.; Corella, D.; Salas-Salvadó, J.; Ros, E.; Covas, M.I.; Fiol, M.; Wärnberg, J.; Arós, F.; Ruíz-Gutiérrez, V.; Lamuela-Raventós, R.M.; et al. Cohort profile: Design and methods of the PREDIMED study. Int. J. Epidemiol. 2012, 41, 377-385. [CrossRef] [PubMed]

23. Fernández-Ballart, J.D.; Piñol, J.L.; Zazpe, I.; Corella, D.; Carrasco, P.; Toledo, E.; Perez-Bauer, M.; Martínez-González, M.A.; Salas-Salvadó, J.; Martín-Moreno, J.M. Relative validity of a semi-quantitative food-frequency questionnaire in an elderly Mediterranean population of Spain. Br. J. Nutr. 2010, 103, 1808-1816. [CrossRef] [PubMed]

24. Martínez-González, M.A.; Fernández-Jarne, E.; Serrano-Martínez, M.; Wright, M.; Gomez-Gracia, E. Development of a short dietary intake questionnaire for the quantitative estimation of adherence to a cardioprotective Mediterranean diet. Eur. J. Clin. Nutr. 2004, 58, 1550-1552. [CrossRef] [PubMed]

25. Elosua, R.; Marrugat, J.; Molina, L.; Pons, S.; Pujol, E. Validation of the Minnesota Leisure Time Physical Activity Questionnaire in Spanish men. Am. J. Epidemiol. 1994, 139, 1197-1209. [CrossRef] [PubMed]

26. Willett, W.C.; Howe, R. Adjustmentfor total energyintake in epidemiologic studies. Am. J. Clin. Nutr. 1997, $65,1220-1228$.

27. Toledo, E.; Hu, F.B.; Estruch, R.; Buil-Cosiales, P.; Corella, D.; Salas-Salvadó, J.; Covas, M.I.; Arós, F.; Gómez-Gracia, E.; Fiol, M.; et al. Effect of the Mediterranean diet on blood pressure in the PREDIMED trial: Results from a randomized controlled trial. BMC Med. 2013, 11, 207. [CrossRef] [PubMed]

28. Martínez-González, M.A.; García-Arellano, A.; Toledo, E.; Salas-Salvadó, J.; Buil-Cosiales, P.; Corella, D.; Covas, M.I.; Schröder, H.; Arós, F.; Gómez-Gracia, E.; et al. A 14-item mediterranean diet assessment tool and obesity indexes among high-risk subjects: The PREDIMED trial. PLoS ONE 2012, 7, e43134. [CrossRef] [PubMed]

29. Razquin, C.; Martinez, J.; Martinez-Gonzalez, M.; Mitjavila, M.T.; Estruch, R.; Marti, A. A 3 years follow-up of a Mediterranean diet rich in virgin olive oil is associated with high plasma antioxidant capacity and reduced body weight gain. Eur. J. Clin. Nutr. 2009, 63, 1387-1393. [CrossRef] [PubMed]

30. Estruch, R.; Martínez-González, M.; Corella, D.; Basora-Gallisá, J.; Ruiz-Gutiérrez, V.; Covas, M.I.; Fiol, M.; Gómez-Gracia, E.; López-Sabater, M.C.; Escoda, R.; et al. Effects of dietary fibre intake on risk factors for cardiovascular disease in subjects at high risk. J. Epidemiol. Community Health 2009, 63, 582-588. [CrossRef] [PubMed]

31. Ibarrola-Jurado, N.; Bulló, M.; Guasch-Ferré, M.; Ros, E.; Martínez-González, M.A.; Corella, D.; Fiol, M.; Wärnberg, J.; Estruch, R.; Román, P.; et al. Cross-Sectional Assessment of Nut Consumption and Obesity, Metabolic Syndrome and Other Cardiometabolic Risk Factors: The PREDIMED Study. PLoS ONE 2013, 8, e57367. [CrossRef] [PubMed] 
32. Bautista-Castaño, I.; Sánchez-Villegas, A.; Estruch, R.; Martínez-González, M.A.; Corella, D.; Salas-Salvadó, J.; Covas, M.I.; Schroder, H.; Alvarez-Pérez, J.; Quilez, J.; et al. Changes in bread consumption and 4-year changes in adiposity in Spanish subjects at high cardiovascular risk. Br. J. Nutr. 2013, 110, 337-346. [CrossRef] [PubMed]

33. Schröder, H. Protective mechanisms of the Mediterranean diet in obesity and type 2 diabetes. J. Nutr. Biochem. 2007, 18, 149-160. [CrossRef] [PubMed]

34. Estruch, R.; Martínez-González, M.A.; Corella, D.; Salas-Salvadó, J.; Fitó, M.; Chiva-Blanch, G.; Fiol, M.; Gómez-Gracia, E.; Arós, F.; Lapetra, J.; et al. Effect of a high-fat Mediterranean diet on bodyweight and waist circumference: A prespecified secondary outcomes analysis of the PREDIMED randomised controlled trial. Lancet Diabetes Endocrinol. 2016, 4, 666-676. [CrossRef]

35. Álvarez-Pérez, J.; Sánchez-Villegas, A.; Díaz-Benítez, E.M.; Ruano-Rodríguez, C.; Corella, D.; Martínez-González, M.Á.; Estruch, R.; Salas-Salvadó, J.; Serra-Majem, L. PREDIMED study investigators. Influence of a Mediterranean dietary pattern on body fat distribution: Results of the PREDIMED-canarias intervention randomized trial. J. Am. Coll. Nutr. 2016, 35, 568-580. [CrossRef] [PubMed]

36. Hughes, L.A.E.; Arts, I.C.W.; Ambergen, T.; Brants, H.A.M.; Dagnelie, P.C.; Goldbohm, R.A.; Van Den Brandt, P.A.; Weijenberg, M.P. Higher dietary flavone, flavonol, and catechin intakes are associated with less of an increase in BMI over time in women: A longitudinal analysis from The Netherlands Cohort Study. Am. J. Clin. Nutr. 2008, 88, 1341-1352. [PubMed]

37. Wang, H.; Wen, Y.; Du, Y.; Yan, X.; Guo, H.; Rycroft, J.A.; Boon, N.; Kovacs, E.M.R.; Mela, D.J. Effects of catechin enriched green tea on body composition. Obesity 2010, 18, 773-779. [CrossRef] [PubMed]

38. Nagao, T.; Komine, Y.; Soga, S.; Meguro, S.; Hase, T.; Tanaka, Y.; Tokimitsu, I. Ingestion of a tea rich in catechins leads to a reduction in body fat and malondialdehyde-modified LDL in men. Am. J. Clin. Nutr. 2005, 81, 122-129. [PubMed]

39. Maki, K.C.; Reeves, M.S.; Farmer, M.; Yasunaga, K.; Matsuo, N.; Katsuragi, Y.; Komikado, M.; Tokimitsu, I.; Wilder, D.; Jones, F.; et al. Green tea catechin consumption enhances exercise-induced abdominal fat loss in overweight and obese adults. J. Nutr. 2009, 139, 264-270. [CrossRef] [PubMed]

40. Stendell-Hollis, N.R.; Thomson, C.A.; Thompson, P.A.; Bea, J.W.; Cussler, E.C.; Hakim, I.A. Green tea improves metabolic biomarkers, not weight or body composition: A pilot study in overweight breast cancer survivors. J. Hum. Nutr. Diet. 2010, 23, 590-600. [CrossRef] [PubMed]

41. Mahler, A.; Steiniger, J.; Bock, M.; Klug, L.; Parreidt, N.; Lorenz, M.; Zimmermann, B.F.; Krannich, A.; Paul, F.; Boschmann, M. Metabolic response to epigallocatechin-3-gallate in relapsing-remitting multiple sclerosis: A randomized clinical trial. Am. J. Clin. Nutr. 2015, 101, 487-495. [CrossRef] [PubMed]

42. Poulsen Morten, M.; Vestergaard Poul, F.; Clasen Berthil, F.; Radko, Y.; Christensen Lars, P.; Stodkilde-Jorgensen, H.; Moller, N.; Jessen, N.; Pedersen, S.B.; Jorgensen, J.O.L. High-dose resveratrol supplementation in obese men: An investigator-initiated, randomized, placebo-controlled clinical trial of substrate metabolism, insulin sensitivity, and body composition. Diabetes 2013, 62, 1186-1195. [CrossRef] [PubMed]

43. Timmers, S.; Konings, E.; Bilet, L.; Houtkooper, R.H.; Van De Weijer, T.; Goossens, G.H.; Hoeks, J.; Van Der Krieken, S.; Ryu, D.; Kersten, S.; et al. Calorie restriction-like effects of 30 days of resveratrol supplementation on energy metabolism and metabolic profile in obese humans. Cell Metab. 2011, 14, 612-622. [CrossRef] [PubMed]

44. Yoshino, J.; Conte, C.; Fontana, L.; Mittendorfer, B.; Imai, S.; Schechtman, K.B.; Gu, C.; Kunz, I.; Rossi Fanelli, F.; Patterson, B.W.; et al. Resveratrol supplementation does not improve metabolic function in nonobese women with normal glucose tolerance. Cell Metab. 2012, 16, 658-664. [CrossRef] [PubMed]

45. Romaguera, D.; Norat, T.; Mouw, T.; May, A.M.; Bamia, C.; Slimani, N.; Travier, N.; Besson, H.; Luan, J.; Wareham, N.; et al. Adherence to the Mediterranean diet is associated with lower abdominal adiposity in European men and women. J. Nutr. 2009, 139, 1728-1737. [CrossRef] [PubMed]

46. Esposito, K.; Kastorini, C.-M.; Panagiotakos, D.B.; Giugliano, D. Mediterranean diet and weight loss: Meta-analysis of randomized controlled trials. Metab. Syndr. Relat. Disord. 2011, 9, 1-12. [CrossRef] [PubMed]

47. Mendez, M.; Popkin, B.M.; Jakszyn, P.; Berenguer, A.; Tormo, M.J.; Sanchéz, M.J.; Quirós, J.R.; Pera, G.; Navarro, C.; Martinez, C.; et al. Adherence to a Mediterranean diet is associated with reduced 3-year incidence of obesity. J. Nutr. 2006, 136, 2934-2938. [PubMed] 
48. Beunza, J.-J.; Toledo, E.; Hu, F.B.; Bes-Rastrollo, M.; Serrano-Martínez, M.; Sánchez-Villegas, A.; Martínez, J.A.; Martínez-González, M.A. Adherence to the Mediterranean diet, long-term weight change, and incident overweight or obesity: The Seguimiento Universidad de Navarra (SUN) cohort. Am. J. Clin. Nutr. 2010, 92, 1484-1493. [CrossRef] [PubMed]

49. Lee, C.M.Y.; Huxley, R.R.; Wildman, R.P.; Woodward, M. Indices of abdominal obesity are better discriminators of cardiovascular risk factors than BMI: A meta-analysis. J. Clin. Epidemiol. 2008, 61, 646-653. [CrossRef] [PubMed]

50. Eguaras, S.; Toledo, E.; Buil-Cosiales, P.; Salas-Salvadó, J.; Corella, D.; Gutierrez-Bedmar, M.; Santos-Lozano, J.M.; Arós, F.; Fiol, M.; Fitó, M.; et al. Does the Mediterranean diet counteract the adverse effects of abdominal adiposity? Nutr. Metab. Cardiovasc. Dis. 2015, 256, 569-574. [CrossRef] [PubMed]

51. Panagiotakos, D.B.; Chrysohoou, C.; Pitsavos, C.; Stefanadis, C. Association between the prevalence of obesity and adherence to the Mediterranean diet: The ATTICA study. Nutrition 2006, 22, 449-456. [CrossRef] [PubMed]

52. Schröder, H.; Mendez, M.A.; Ribas-Barba, L.; Covas, M.I.; Serra-Majem, L. Mediterranean diet and waist circumference in a representative national sample of young Spaniards. Int. J. Pediatr. Obes. 2010, 5, 516-519. [CrossRef] [PubMed]

53. Nagao, T.; Hase, T.; Tokimitsu, I. A green tea extract high in catechins reduces body fat and cardiovascular risks. Obesity 2007, 15, 1473-1483. [CrossRef] [PubMed]

54. Cai, L.; Han, X.; Qi, Z.; Li, Z.; Zhang, Y.; Wang, P.; Liu, A. Prevalence of overweight and obesity and weight loss practice among Beijing adults, 2011. PLoS ONE 2014, 9, e98744. [CrossRef] [PubMed]

55. Wang, Y.; Beydoun, M.A. The obesity epidemic in the United States-Gender, age, socioeconomic, racial/ethnic, and geographic characteristics: A systematic review and meta-regression analysis. Epidemiol. Rev. 2007, 29, 6-28. [CrossRef] [PubMed]

56. Williams, R.L.; Wood, L.G.; Collins, C.E.; Callister, R. Effectiveness of weight loss interventions - is there a difference between men and women: A systematic review. Obes. Rev. 2015, 16, 171-186. [CrossRef] [PubMed]

57. Gross, G.; Jacobs, D.M.; Peters, S.; Possemiers, S.; Van Duynhoven, J.; Vaughan, E.E.; Van De Wiele, T. In vitro bioconversion of polyphenols from black tea and red wine/grape juice by human intestinal microbiota displays strong interindividual variability. J. Agric. Food Chem. 2010, 58, 10236-10246. [CrossRef] [PubMed]

58. Stevens, J.; Katz, E.G.; Huxley, R.R. Associations between gender, age and waist circumference. Eur. J. Clin. Nutr. 2010, 64, 6-15. [CrossRef] [PubMed]

59. Jo, J.; Gavrilova, O.; Pack, S.; Jou, W.; Mullen, S.; Sumner, A.E.; Cushman, S.W.; Periwal, V. Hypertrophy and/or hyperplasia: Dynamics of adipose tissue growth. PLoS Comput. Biol. 2009, 5, e1000324. [CrossRef] [PubMed]

60. Carpéné, C.; Gomez-Zorita, S.; Deleruyelle, S.; Carpéné, M.A. Novel strategies for preventing diabetes and obesity complications with natural polyphenols. Curr. Med. Chem. 2015, 22, 150-164. [CrossRef] [PubMed]

61. Szkudelska, K.; Szkudelski, T. Resveratrol, obesity and diabetes. Eur. J. Pharmacol. 2010, 635, 1-8. [CrossRef] [PubMed]

62. Tsuda, T. Dietary anthocyanin-rich plants: Biochemical basis and recent progress in health benefits studies. Mol. Nutr. Food Res. 2012, 56, 159-170. [CrossRef] [PubMed]

63. Wright, O.R.L.; Netzel, G.A.; Sakzewski, A.R. A randomized, double-blind, placebo-controlled trial of the effect of dried purple carrot on body mass, lipids, blood pressure, body composition, and inflammatory markers in overweight and obese adults: The QUENCH Trial. Can. J. Physiol. Pharmacol. 2013, 91, 480-488. [CrossRef] [PubMed]

64. Zhu, Y.; Xia, M.; Yang, Y.; Liu, F.; Li, Z.; Hao, Y.; Mi, M.; Jin, T.; Ling, W. Purified anthocyanin supplementation improves endothelial function via NO-cGMP activation in hypercholesterolemic individuals. Clin. Chem. 2011, 57, 1524-1533. [CrossRef] [PubMed]

65. Yao, L.H.; Jiang, Y.M.; Shi, J.; Tomás-Barberán, F.A.; Datta, N.; Singanusong, R.; Chen, S.S. Flavonoids in food and their health benefits. Plant Foods Hum. Nutr. 2004, 59, 113-122. [CrossRef] [PubMed]

66. Tresserra-Rimbau, A.; Medina-Remón, A.; Pérez-Jiménez, J.; Martínez-González, M.A.; Covas, M.I.; Corella, D.; Salas-Salvadó, J.; Gómez-Gracia, E.; Lapetra, J.; Arós, F.; et al. Dietary intake and major food sources of polyphenols in a Spanish population at high cardiovascular risk: The PREDIMED study. Nutr. Metab. Cardiovasc. Dis. 2013, 23, 953-959. [CrossRef] [PubMed] 
67. Andersen, C.; Rayalam, S.; Della-Fera, M.A.; Baile, C.A. Phytochemicals and adipogenesis. BioFactors 2010, 36, 415-422. [CrossRef] [PubMed]

68. Galleano, M.; Calabro, V.; Prince, P.D.; Litterio, M.C.; Piotrkowski, B.; Vazquez-Prieto, M.A.; Miatello, R.M.; Oteiza, P.I.; Fraga, C.G. Flavonoids and metabolic syndrome. Ann. N. Y. Acad. Sci. 2012, 1259, 87-94. [CrossRef] [PubMed]

69. Kobayashi, M.; Kawano, T.; Ukawa, Y.; Sagesaka, Y.M.; Fukuhara, I. Green tea beverages enriched with catechins with a galloyl moiety reduce body fat in moderately obese adults: A randomized double-blind placebo-controlled trial. Food Funct. R. Soc. Chem. 2016, 7, 498-507. [CrossRef] [PubMed]

70. Brüll, V.; Burak, C.; Stoffel-Wagner, B.; Wolffram, S.; Nickenig, G.; Müller, C.; Langguth, P.; Alteheld, B.; Fimmers, R.; Naaf, S.; et al. Effects of a quercetin-rich onion skin extract on $24 \mathrm{~h}$ ambulatory blood pressure and endothelial function in overweight-to-obese patients with (pre-)hypertension: A randomised double-blinded placebo-controlled cross-over trial. Br. J. Nutr. 2015, 114, 1263-1277. [CrossRef] [PubMed]

71. Tresserra-Rimbau, A.; Guasch-Ferre, M.; Salas-Salvado, J.; Toledo, E.; Corella, D.; Castaner, O.; Guo, X.; Gomez-Gracia, E.; Lapetra, J.; Aros, F.; et al. Intake of total polyphenols and some classes of polyphenols is inversely associated with diabetes in elderly people at high cardiovascular disease risk. J. Nutr. 2016, 146, 767-777. [CrossRef] [PubMed]

72. Wing, R.; Lang, W.; Wadden, T.; Safford, M.; Knowler, W.; Bertoni, A.; Hill, J.; Brancati, F.; Peters, A.; Wagenknecht, L. Benefits of modest weight loss in improving cardiovascular risk factors in overweight and obese individuals with type 2 diabetes. Diabetes Care 2011, 34, 1481-1486. [CrossRef] [PubMed]

73. Guo, X.; Tresserra-Rimbau, A.; Estruch, R.; Martínez-González, M.A.; Medina-Remón, A.; Castañer, O.; Corella, D.; Salas-Salvadó, J.; Lamuela-Raventós, R.M. Effects of polyphenol, measured by a biomarker of total polyphenols in urine, on cardiovascular risk factors after a long-term follow-up in the PREDIMED study. Oxid. Med. Cell. Longev. 2016, 2016, 2572606. [CrossRef] [PubMed]

74. Nagasako-Akazome, Y.; Kanda, T.; Ohtake, Y.; Shimasaki, H.; Kobayashi, T. Apple polyphenols influence cholesterol metabolism in healthy subjects with relatively high body mass index. J. Oleo Sci. 2007, 56, 417-428. [CrossRef] [PubMed]

75. Klein, S.; Allison, D.; Heymsfield, S.; Kelley, D.; Leibel, R.; Nonas, C.; Kahn, R.E.A.; Leibe, R. Waist circumference and cardiometabolic risk: A consensus statement from Shaping America's Health: Association for weight management and obesity prevention. Am. J. Clin. Nutr. 2007, 85, 1197-1202. [PubMed]

76. Hervert-Hernández, D.; Goñi, I. Contribution of beverages to the intake of polyphenols and antioxidant capacity in obese women from rural Mexico. Public Health Nutr. 2012, 15, 6-12. [CrossRef] [PubMed]

(C) 2017 by the authors. Licensee MDPI, Basel, Switzerland. This article is an open access article distributed under the terms and conditions of the Creative Commons Attribution (CC BY) license (http:/ / creativecommons.org/licenses/by/4.0/). 